\title{
In Situ Mapping of Radionuclides in Subsurface and Surface Soils: 1994 Summary Report
}
A. J. Schilk
C. W. Hubbard
M. A. Knopf
K. H. Abel

April 1995

Prepared by the the U.S. Department of Energy under Contract DE-AC06-76RLO 1830

Funded by the Office of Technology Development, within the U.S. Department of Energy's Office of Environmental Management, under the Uranium in Soils Integrated Demonstration

Pacific Northwest Laboratory Operated for the U.S. Department of Energy by Battelle Memorial Institute 


\title{
DISCLAIMER
}

This report was prepared as an account of work sponsored by an agency of the United States Government. Neither the United States Government nor any agency thereof, nor Battelle Memorial Institute, nor any of their employees, makes any warranty, expressed or implied, or assumes any legal liability or responsibility for the accuracy, completeness, or usefulness of any information, apparatus, product, or process disclosed, or represents that its use would not infringe privately owned rights. Reference herein to any specific commercial product, process, or service by trade name, trademark, manufacturer, or otherwise does not necessarily constitute or imply its endorsement, recommendation, or favoring by the United States Government or any agency thereof, or Battelle Memorial Institute. The views and opinions of authors expressed herein do not necessarily state or reflect those of the United States Government or any agency thereof.

\author{
PACIFIC NORTHWEST LABORATORY \\ operated by \\ BATTELLE MEMORIAL INSTITUTE \\ for the \\ UNITED STATES DEPARTMENT OF ENERGY \\ under Contract DE-AC06-76RLO 1830
}

Printed in the United States of America

Available to DOE and DOE contractors from the

Office of Scientific and Technical Information; P.O. Box 62, Oak Ridge, IN 37831;

prices available from (615) 576-8401. FTS 626-8401.

Available to the public from the National Technical Information Service,

U.S. Department of Commerce, 5285 Port Royal Rd., Springfield, VA 22161. 
PNL-10484

UC-606

\title{
In Situ Mapping of Radionuclides in Subsurface and Surface Soils: 1994 Summary Report
}

\author{
A.J. Schilk \\ C.W. Hubbard \\ M.A. Knopf \\ K.H. Abel
}

April 1995

Prepared for the U.S. Department of Energy under Contract DE-AC06-76RLO 1830

This work was funded by the Office of Technology Development, within the U.S. Department of Energy's Office of Environmental Management, under the Uranium in Soils Integrated Demonstration

Pacific Northwest Laboratory

Richland, Washington 99352 


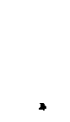

. 


\section{Executive Summary}

Uranium production and support facilities at several DOE sites occasionally caused local contamination of some surface and subsurface soils. The thorough cleanup of these sites is a major public concern and a high priority for the U.S. Department of Energy, but before any effective remedial protocols can be established, the three-dimensional distributions of target contaminants must be characterized.

Traditional means of measuring radionuclide activities in soil are cumbersome, expensive, timeconsuming, and often do not accurately reflect conditions over very large areas. New technologies must be developed, or existing ones improved, to allow cheaper, faster, and safer characterization of radionuclides in soils at these sites.

The Pacific Northwest Laboratory (PNL) was tasked with adapting, developing, and demonstrating technologies to measure uranium in surface and subsurface soils. In partial completion of this effort, PNL developed an improved in situ gamma-ray spectrometry system to satisfy the technical requirements.

This document summarizes fiscal-year 1994 efforts at PNL to fulfill requirements for TTP \#32 I 103 (project \#19307). These requirements included (a) developing a user-friendly software package for reducing field-acquired gamma-ray spectra, (b) constructing an improved data-acquisition hardware system for use with high-purity germanium detectors, (c) ensuring readiness to conduct field mapping exercises as specified by the sponsor, (d) evaluating the in situ gamma-ray spectrometer for the determination of uranium depth distribution, and (e) documenting these efforts 



\title{
Acknowledgements
}

This work was prepared with the support of the following contributors:

\author{
Headquarters: $\quad$ Office of Technology Development \\ Uranium in Soils Integrated Demonstration \\ Michael Malone \\ Operations Office: Richland Operations Office \\ Technology Development Division \\ Deborah E. Trader, Technical Program Officer \\ Contractor: \\ Pacific Northwest Laboratory \\ Environmental Management Directorate \\ Steven C. Slate, Technical Program Manager
}


. 


\section{Contents}

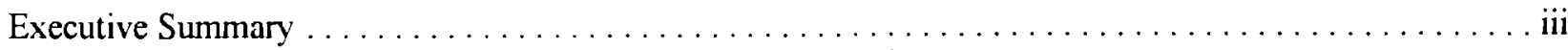

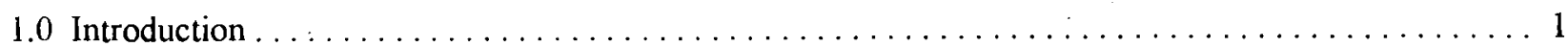

2.0 Technological Theory, System Description, and Methodology $\ldots \ldots \ldots \ldots \ldots \ldots \ldots \ldots \ldots$

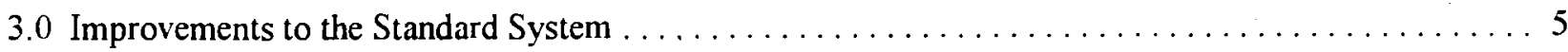

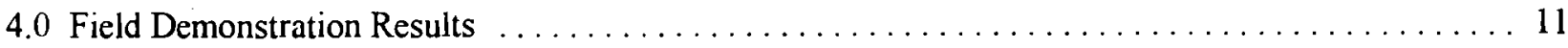

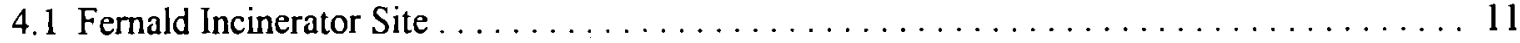

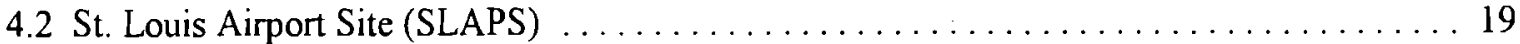

5.0 System Capability for Depth-Distribution Determination of Contaminants $\ldots \ldots \ldots \ldots \ldots \ldots \ldots 21$

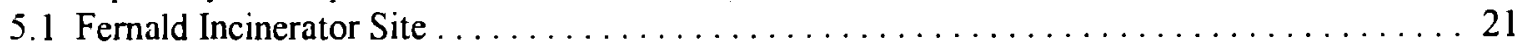

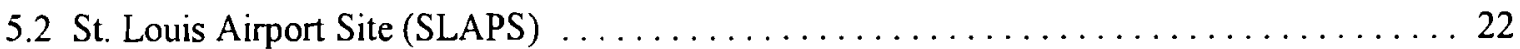

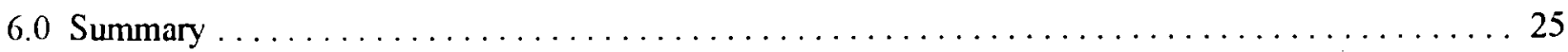

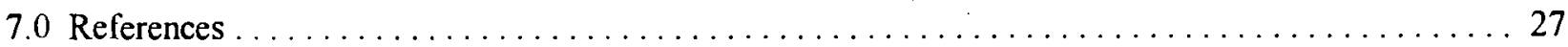

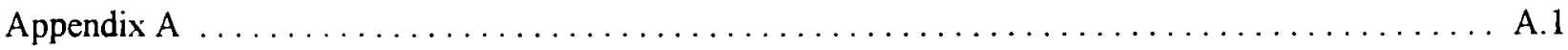




\section{Figures}

3.1 Simplified diagram showing the standard in situ gamma-ray spectrometry system and its

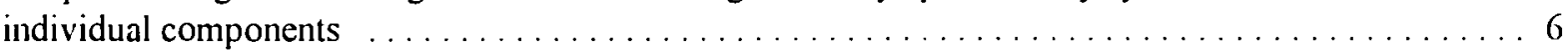

3.2 Simplified diagram of the improved in situ gamma-ray spectrometry system $\ldots \ldots \ldots \ldots \ldots \ldots 7$

3.3 Configuration of the individual components in the miniature data-acquisition hardware package (courtesy of the Los Alamos National Laboratory)

3.4 Interface schematic for the miniature data-acquisition hardware package (courtesy of the Los Alamos National Laboratory)

4.1 Base map for the field demonstration area, directly north of the Fernald incinerator area. Sample sites are indicated by solid circles; southernmost row of data points (discontinuous due to the presence of the exclusion area) are equivalent to those found immediately north of the northern fenceline surrounding the incinerator site as identified in Schilk et al. 1993a. The approximate location of the actual incinerator stack is: easting $=+50$ feet, northing $=-50$ feet. Crosses indicate samples that were cored for depth-distribution analyses

4.2 Uranium surface activity contour map based on a geostatistical analysis of the $100-\mathrm{cm}$ in situ gamma-ray' spectrometry measurements

4.3 Uranium surface activity contour map based on a geostatistical analysis of the $15-\mathrm{cm}$ in situ gamma-ray spectrometry measurements. Qualitatively, these results are consistent with Figure 4.2. See text for explanation of quantitative differences

4.4 Uranium surface activity contour map based on a geostatistical analysis of the hand sample/ laboratory analysis measurements. Qualitatively, these results are consistent with Figure 4.2. See text for explanation of quantitative differences

4.5 Correlation plot comparing the $100-\mathrm{cm}$ and $15-\mathrm{cm}$ in situ gamma-ray measurements. The correlation coefficient is moderate, indicating that the results tend to be mutually supportive $\ldots \ldots 16$

4.6 Correlation plot comparing the $100-\mathrm{cm}$ in situ gamma-ray measurements and the hand sample/ laboratory analysis measurements

4.7 Corrclation plot comparing the $15-\mathrm{cm}$ in situ gamma-ray measurements and the hand sample/ laboratory analysis measurements

5.1 Mcasured uranium depth distributions for the five cored samples (refer to Figure 4.1 for locations) Legend: circle - NW point, square - NE point, triangle (down) - SW point, diamond - SE point, triangle (up) - central point

5.2 Plot of effective uranium activity as a function of photon energy when a uniformly distributed source was assumed. Increasing effective activity with higher photon energies impliesa buried source 


\section{Tables}

Table 5.1 Calculated intensity ratios for selected gamma rays from the ${ }^{238} \mathrm{U}$ decay chain $\ldots \ldots \ldots 21$

Table 5.2 Observed gamma-ray intensity ratios for five locations north of the Fernald incinerator

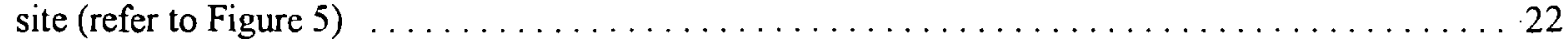





\subsection{Introduction}

To establish cleanup requirements at nuclear waste sites across the nation, the U.S. Department of Energy's (DOE's) Director of Environmental Restoration and Waste Management initiated the Office of Technology Development (OTD) and charged it with the evaluation of existing and projected remediation and characterization technologies. The OTD created the Integrated Demonstration (ID) program and tasked it with investigating the efficacy, cost effectiveness, risk-reduction potential, site applicability, and regulatory/public acceptance of proposed technologies. The intent is to transfer technologies to other DOE operations or the private sector. The Uranium in Soils ID was created to evaluate and compare the efficiency, versatility, and economic viability of specific technologies used to characterize and remediate uraniumcontaminated soils.

Uranium production and support facilities at several DOE sites occasionally caused local contamination of some surface and subsurface soils. Such contamination commonly occurs within waste burial sites, cribs, pond bottom sediments, and areas surrounding waste tanks or uranium scrap, ore, tailing, and slag heaps. The thorough cleanup of these sites is a major public concern and a high priority for the DOE, but before any cffective remedial protocols can be established, the three-dimensional distributions of target contaminants must be characterized. Unfortunately, traditional means of measuring radionuclide activities in soil (e.g., grab sampling followed by laboratory analyses) are cumbersome, expensive, time-consuming, and often do not accurately reflect conditions over very large areas. New technologies must be developed, or existing ones improved, to allow cheaper, faster, and safer characterization of radionuclides in soils at these sites.

The Pacific Northwest Laboratory (PNL) was tasked with adapting, developing, and demonstrating technologies to measure uranium in surface and subsurface soils. In partial completion of this effort, PNL (with the collaboration of scientists and engineers at the Los Alamos National Laboratory and EG\&G's Rocky Flats site) developed an improved in situ gamma-ray spectrometry system. The improved system is composed of a conmercially available high-purity germanium detector, a miniaturized electronic support package, and a user-friendly software package for evaluating the field-acquired gamma-ray spectra. This combination can facilitate rapid and efficient characterization of radionuclide-contaminated soils by making gamma-ray spectrometry technology accessible to operators with minimal training or experience in this tcchnology. Significant savings of total measurement time, manpower, and overall equipment costs are anticipated for characterization and remediation operations conducted on soils contaminated with gamma-rayemilting radionuclides.

This paper summarizes the efforts to develop the system during the 1994 fiscal year, and discusses results from field demonstrations of the improved gamma-ray detector system. 



\subsection{Technological Theory, System Description, and Methodology}

For decades, gamma-ray spectrometry has been used in laboratories around the world to characterize efficiently a multitude of radionuclides. This technology's many advantages as an analytical tool for environmental monitoring include its selective high resolution (enabling precise, quantitative, multiradioisotopic characterization), relatively easy transportability, and rapid data reduction to provide essentially real-time results in the field. Nonetheless, its use with regard to the quantification of radionuclide content in soils has been somewhat limited. Regulatory approval to use this technology in the field has not yet been granted, although an international panel of radiation-detection experts has recently declared it is a "rapid and sound method for the determination of radionuclide activities in the environment" (ICRU 1993).

A high-purity germanium gamma-ray detector, suspended above the ground in an uncollimated configuration, is sensitive to surface and shallow subsurface activity originating from tens to hundreds of square meters, and effectively averages any horizontal heterogeneities that may exist within its field of view. Although in theory this type of system would monitor complete $2 \pi$-space (i.e., from horizon to horizon), the actual depth and expanse of contamination detected is a function of the photon energy. For example, approximately $80 \%$ to $90 \%$ of the total observed fluence originates within a 10 -meter radius from the detector due to geometry factors and attenuation for most soil distributions and gamma energies exceeding about $500 \mathrm{keV}$. A rather large surface area may be characterized, therefore, in a relatively short period of time by establishing a triangular map grid with a 20 -meter intersample spacing.

In practice, photons (i.e., gammas and $x$-rays) that are incident upon the sensor crystal lead to ionizations and excitations of myriad germanium atoms, leading to the generation of an equivalent number of oppositely charged species, namely, electrons (negative) and electron "holes" (positive). A strong electrical potential (generally on the order of a few thousand volts) sweeps the charge-carrying species to opposing regions of the germanium crystal, where they are subsequently collected and quantified. The greater the amount of total energy deposited from an isolated event (e.g., due to the attenuation of a lone photon), the larger the total charge collected. By observing the magnitudes of individual charge pulses one may determine the various energies of the photons undergoing complete absorption in the crystal. The specific photon energies serve as identifiers for various radionuclides of interest; e.g., 1.461-MeV gamma rays originate from the spontaneous decay of ${ }^{40} \mathrm{~K}, 0.662-\mathrm{MeV}$ gammas represent ${ }^{137} \mathrm{Cs}$, and $1.001-\mathrm{MeV}$ gammas are an indication of the presence of ${ }^{234 m} \mathrm{~Pa}$ (a daughter of ${ }^{238} \mathrm{U}$ ). This, combined with the fact that the total number of equivalent pulses per unit time is an indication of the actual amount of source material present, allows the qualitative and quantitative measurements of target radionuclides (such as uranium) to be determined in the field.

The ${ }^{238} \mathrm{U}$ decay chain is as follows:

${ }^{238} \mathrm{U}\left(4.47 \times 10^{9} \mathrm{y}\right) \rightarrow{ }^{23+} \mathrm{Th}(24.1 \mathrm{~d}) \rightarrow{ }^{234 \mathrm{~m}} \mathrm{~Pa}(1.18 \mathrm{~m}) \rightarrow{ }^{234} \mathrm{U}\left(2.45 \times 10^{5} \mathrm{y}\right) \rightarrow$
${ }^{230} \mathrm{Th}\left(7.54 \times 10^{4} \mathrm{y}\right) \rightarrow{ }^{226} \mathrm{Ra}\left(1.60 \times 10^{3} \mathrm{y}\right) \rightarrow($ radon+daughters $) \rightarrow{ }^{206} \mathrm{~Pb}$ (stable).

If secular equilibrium may be assumed (i.e., if no fractionation between ${ }^{238} \mathrm{U}$ and ${ }^{234} \mathrm{Th}$ or ${ }^{234 \mathrm{~m} P a}$ has occurred within the past few months), then the decay rates of the latter radionuclides are essentially equivalent to that of uranium. This technology can also be used to estimate uranium depth distributions. This is accomplished by comparing count rates at various photon energies for ${ }^{234} \mathrm{Th}$ and ${ }^{234 \mathrm{mPa}}$. Due to the differing attenuation 
properties of these photons, one may compare their observed intensities with those expected from various vertical distributions and determine, to a first-order magnitude, the behavior of the contaminant in the upper few centimeters of soil. This ability is limited, however, by the necessary assumption that the vertical distribution is consistent in the horizontal dimension, which may not always be the case.

The improved in situ spectrometry system is centered around a commercial germanium-crystal-based gamma-ray sensor. The specific detector was chosen for its enhanced sensitivity to low-energy photons, which facilitates characterization of low-energy gammas from ${ }^{234} \mathrm{Th}$. The germanium crystal must be kept cold during normal operation to minimize the electronic noise inherent to this type of sensor; liquid nitrogen in a reservoir attached directly to the crystal housing cools the crystal. The sensor package consists of a cylindrical crystal housing (9-cm diameter $\times 28-\mathrm{cm}$ length) surmounted by a reservoir of the same shape (22$\mathrm{cm}$ diameter $\mathrm{x} 28 \mathrm{~cm}$ ). This package is suspended above the ground surface on a sturdy tripod, such that the detector endcap faces the soil. The total weight of these components is somewhat less than $15 \mathrm{~kg}$.

The gamma-ray detector must be calibrated by different radioactive sources in various orientations. Sealed ${ }^{60} \mathrm{Co},{ }^{137} \mathrm{Cs}$, ${ }^{241} \mathrm{Am}$, and ${ }^{152} \mathrm{Eu}$ sources are normally placed 1 meter from the endcap at a number of angles to the detector face to determine the angular response. Total gamma-ray counts for each source/orientation combination are compared with actual source strengths, and detector efficiencies for each scenario are calculated. This detector-specific information is combined with data that indicate the expected gamma-ray fluxes originating from soils exhibiting various vertical radionuclide distributions. These data are easily calculated using radiation theory (e.g., attenuation coefficients) and simple mathematics, and are entirely independent of the detector being calibrated.

This detector-specific and detector-independent combination generates unique calibration factors with units of $\mathrm{pCi} / \mathrm{g} /$ counts per second for all energies (or radionuclides) of interest. The calibration is accurate only for uniform vertical distributions of contaminants, since the actual $\mathrm{pCi} / \mathrm{g}$ for a non-uniform distribution is a direct function of the depth. Normally, one may assume a uniform distribution, despite evidence to the contrary, and obtain the average $\mathrm{pCi} / \mathrm{g}$ for the soil. For example, to determine the specific activity of ${ }^{238} \mathrm{U}$ in soil one would obtain an in situ gamma-ray spectrum, determine the total number of counts in the $1.001-\mathrm{MeV}$ ${ }^{234 m}$ Pa peak per unit time (i.e., the total counts per second), multiply this parameter by the unique calibration factor for this energy, and thereby obtain the total activity of ${ }^{238} U$ per gram of soil (by doubling this value, one obtains the $\mathrm{pCi} / \mathrm{g}$ of total uranium). In practice, this procedure is performed for multiple photons from the uranium decay chain, and these results are combined and compared to generate data that are internally consistent. 


\subsection{Improvements to the Standard System}

With regard to the supporting data-acquisition hardware, available equipment (Figure 3.1) consists of a remote system containing all of the electronic components necessary for proper detector operation; such a unit requires external AC power and includes a bias supply for the detector, an amplifier to shape and amplify the detector output signal, an analog-to-digital convertor to translate this signal into a usable form, and a memory buffer for storing the acquired data. The overall dimensions of this system are approximately $30 \mathrm{~cm} \times 30 \mathrm{~cm}$ $\times 30 \mathrm{~cm}$. Data display, acquisition control, and data reduction are performed by a standard laptop computer, which receives output from the hardware unit and includes a standard gamma-ray peak-search program. The problems associated with this system include the need for two operators to ensure safe deployment in the field (one to advance the detector and another to manipulate the computer and electronics), the dragging of lengthy cables through soils that may be contaminated, and the lack of a technology-specific data-reduction software for in situ field applications.

A number of improvements have been made to the standard spectrometry package in the past year to enhance its field maneuverability, ease of use for non-spectroscopists, and safe operation. In particular, miniaturized electronics have been developed that eliminate the need for bulky power supplies, signal amplifiers, analog-to-digital convertors, computers, etc. and lengthy power/signal cable spans (Figure 3.2). In this configuration, all of the electronics are condensed into an $10-\mathrm{cm} \times 20-\mathrm{cm} \times 9-\mathrm{cm}$ package (Halbig et al. $1993 ; 1994$ ) that attaches directly to the liquid-nitrogen reservoir and contains its own battery-supplied power. System control and data display are maintained by a local palmtop computer, also mounted to the liquid-nitrogen reservoir, and data reduction occurs either remotely or in situ at the operator's (or field supervisor's) convenience.

The improved electronic system, developed at the Los Alamos National Laboratory, consists of a basic multichannel analyzer, its supporting hardware and firmware, and a software library that serves as an interface between external programs and the analyzer itself. The unit is powered internally by a removable, 12-V (2.2 A-h) nickel-metal-hydride computer battery that has a lifetime of 3 to 4 hours (depending on the load created by the detector in use). The hardware (Figure 3.3) consists of a high-performance amplifier with selectable time constants, fine and coarse gain adjustments, pulse pile-up rejection, deadtime correction, and active, gated baseline restoration. The analog-to-digital convertor is a 100-Mhz Wilkinson type with 512 (normally used for $\mathrm{NaI}[\mathrm{Tl}]$ applications) and 4096 channel conversion gains. The processor board has two serial ports for data transfer and system control (16-bit $1 / 0$ ), nonvolatile EEPROM for parameter storage, and a flash disk for program storage. The power supply and bias boards provide both low-voltage and bias power to the gamma-ray sensor as necessary for proper operation (refer to Halbig et al. 1993 for additional component features).

The system firmware controls the hardware, responding to commands transferred through the serial interface from the palmtop computer (which contains the data-acquisition software code). The firmware's accompanying command set includes controls for the individual hardware functions (including diagnostics) and the ability to query and read back resident gamma-ray measurement parameters (e.g., region-of-interest operations and centroid calculations). The serial commands and their responses are ASCII strings, except in the case of transferred spectral data, which may be either ASCII or binary. Although the serial interface is slower than a parallel or bus interface, it was chosen because of its wide use, availability, and the fact that the firmware has been specifically designed to minimize the need for rapid data-transfer rates. 


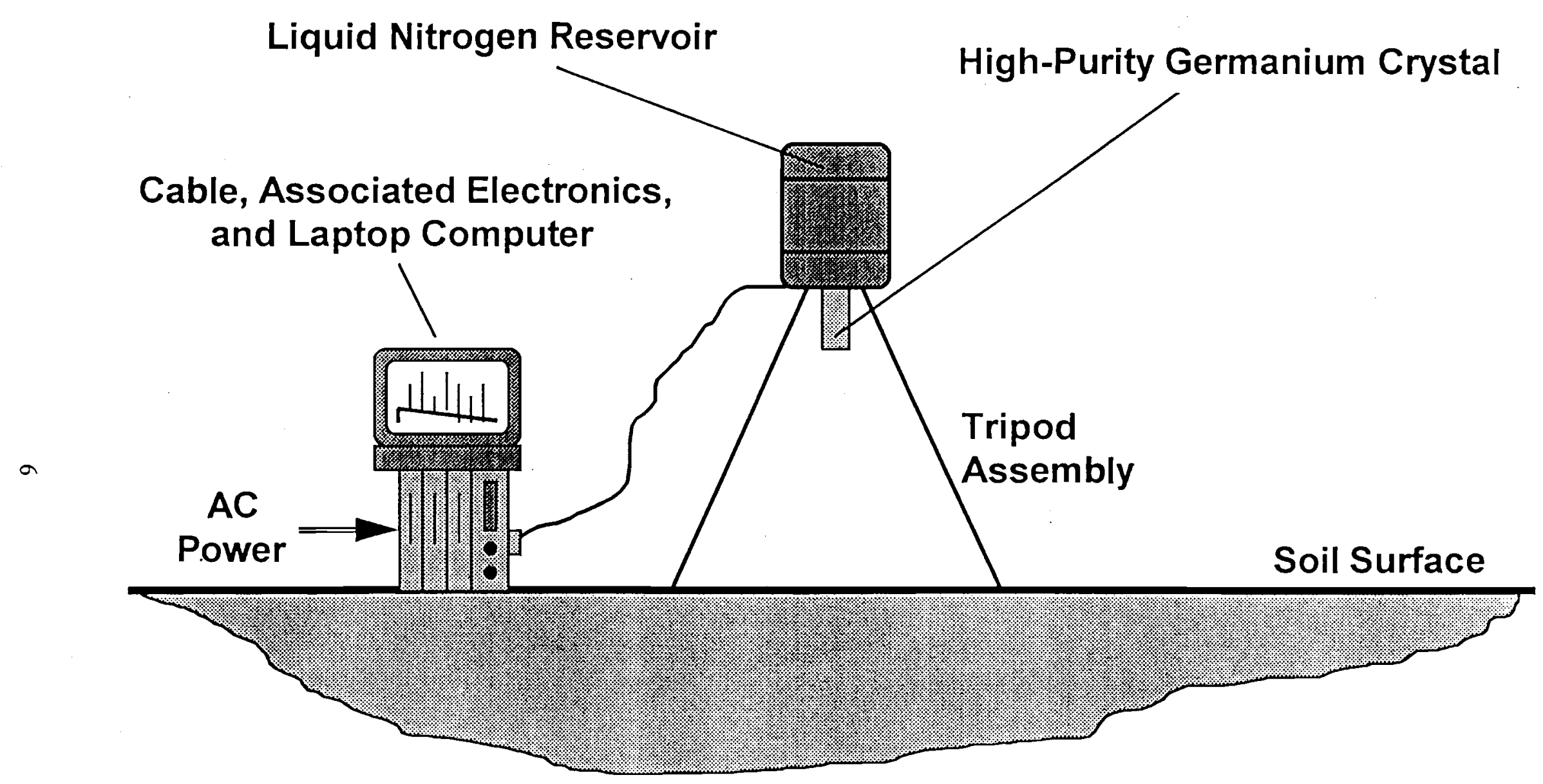

Figure 3.1. Simplified diagram showing the standard in situ gamma-ray spectrometry system and its individual components. 


\section{Liquid Nitrogen Reservoir}

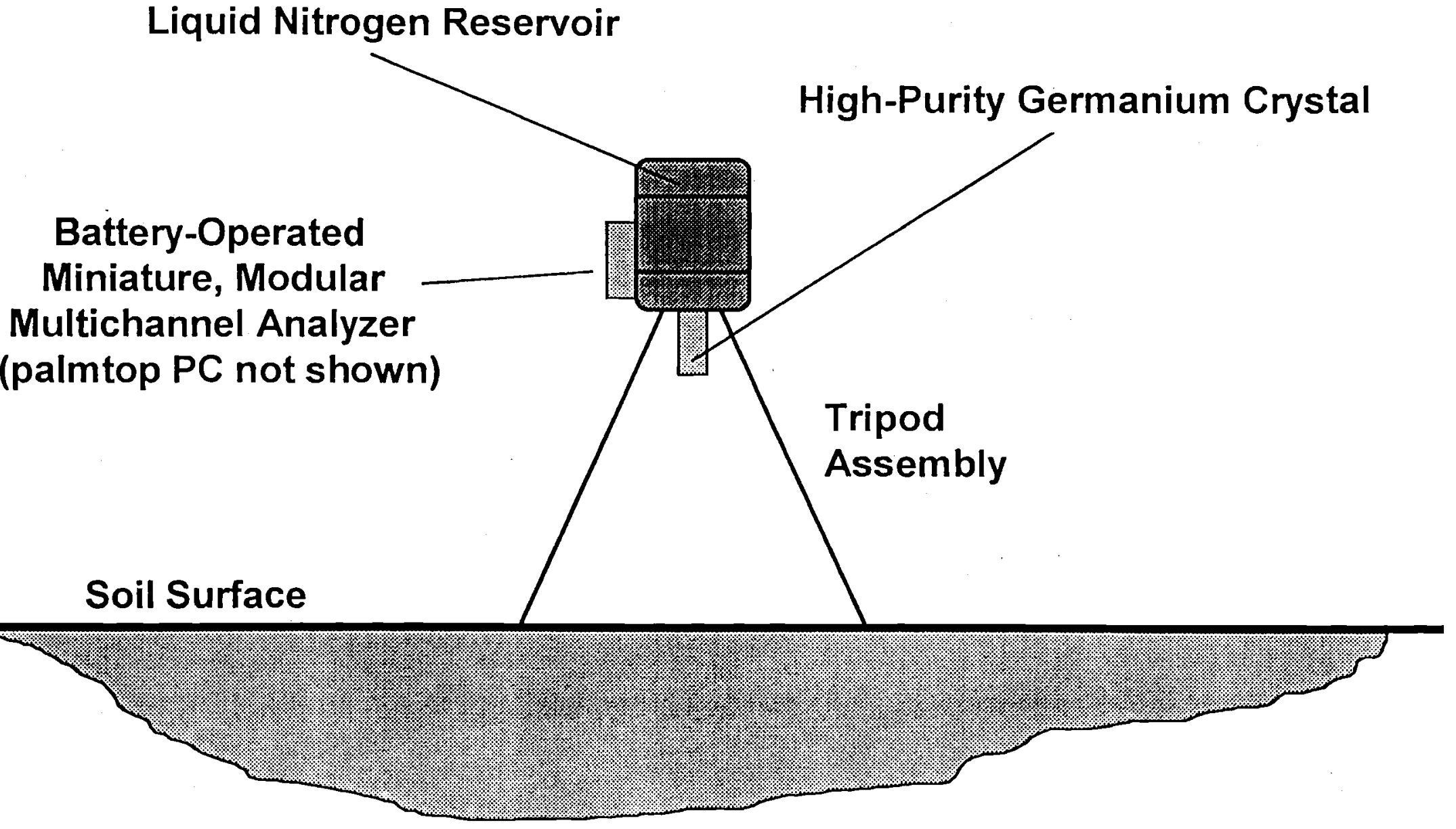

Figure 3.2. Simplified diagram of the improved in situ gamma-ray spectrometry system. 


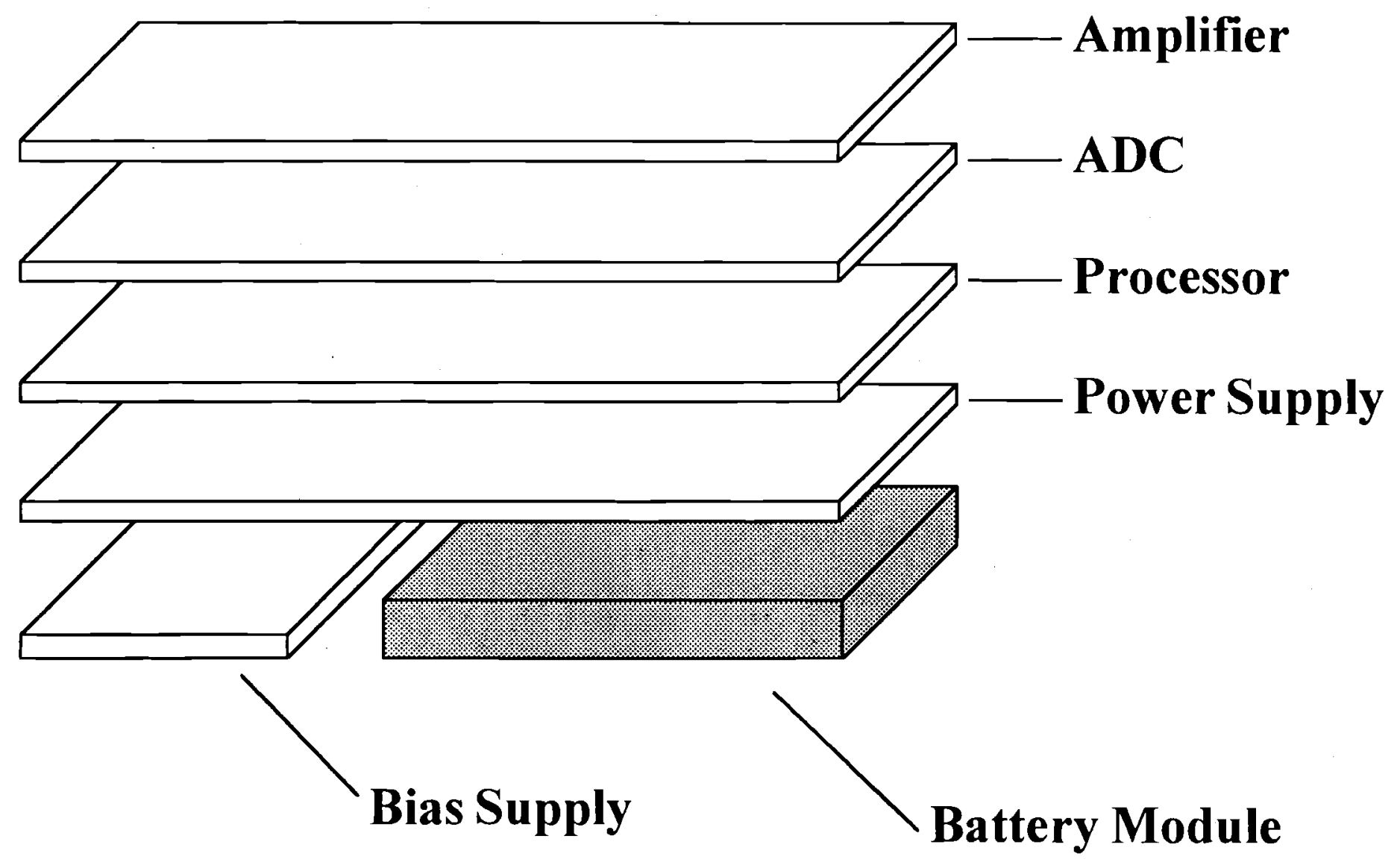

Figure 3.3. Configuration of the individual components in the miniature data-acquisition hardware package (courtesy of the Los Alamos National Laboratory). 
This combination of palmtop-computer-resident acquisition software, serial interface, and electronic hardware/firmware (Figure 3.4) is ideally suited to meet the need for an intelligent, easily maneuverable, single-operator field system for characterizing radionuclide content in soils. The operator simply places the tripod-mounted system over the target location, keys in a unique sample identifier and initiates the data acquisition via the local palmtop computer, awaits the completion of the acquisition phase, moves the unit to the next location, and repeats the process. Individual sample results are automatically stored on the computer-resident flash disk, which may be quickly replaced and transferred at any time to the field supervisor for data reduction and archiving while field measurements continue. Future improvements to this system include the incorporation of a global-positioning package (e.g., utilizing a commercially available interface) to eliminate the need for individual sample identifiers and preliminary field surveys to pinpoint measurement locations.

Although application-specific software programs for controlling the acquisition of gamma-ray spectra are numerous and readily obtained, no reliable data-reduction software package is commercially available for this technology. PNL developed a code with a user-friendly graphical interface (using a mousedriven desktop environment) that facilitates rapid and efficient data reduction of field-acquired gamma-ray spectra by non-specialists.

This program, referred to as "Sassy" for Spectral Analysis Software for Environmental Applications (SASEA), can accept digitized gamma-ray spectra in any number of various proprietary formats and allows either manual analyses of individual spectra or an automatic, multi-spectral data-reduction option to minimize redundancy and save time. The operator may also perform pre-reduction smoothing of the raw spectra (e.g., by a cubic spline) to evaluate the effects on the final data. Tabulated spectrum results may be sent directly to the screen, a printer, a specified file, or a combination thereof. A condensed version of these results, containing only the activity levels of the most prominent radionuclides observed in the spectrum, may be saved or viewed. Alternatively, a complete tabulation of all identified peaks, including net and gross counts, activity levels, counting errors, etc., may be archived in its entirety.

The SASEA code is equipped with an interactive spectrum-plotting capability that lets the user view individual spectra for observing background and peak behavior, manually verify results, interpret abnormal conditions, and evaluate the efficacy of the data-reduction process. For example, the operator may click or drag the mouse to zoom in on regions of interest in any spectrum, or zoom out to again to obtain a fullspectrum view.

This package also provides a thorough options environment for controlling and optimizing the datareduction process, including numerous peak libraries to choose from (natural isotopes only, fission and activation products, cosmic-induced species, etc.); a means of indicating the source-to-detector distance (to allow placement of the sensor nearer the ground surface in a pseudo-collimated configuration) and source areal extent (e.g., point source versus extended source); the identification of detector-specific calibration and efficiency files (in the case of multi-detector configurations); choice of activity units ( $\mathrm{Ci}$ versus $\mathrm{Bq}$ ); and sensitivity options for the peak-search and peak-find processes.

The SASEA program is evolving and modifications are being incorporated on a regular basis. Nonetheless, the versatility and usability of this package far exceed that of any comparable code on the market today, and discussions have been initiated with a number of potential industrial partners for the conmercialization of this code. Appendix A provides a summary of the operational screens currently available in this software package, and brief descriptions of the various options that exist for each screen. 


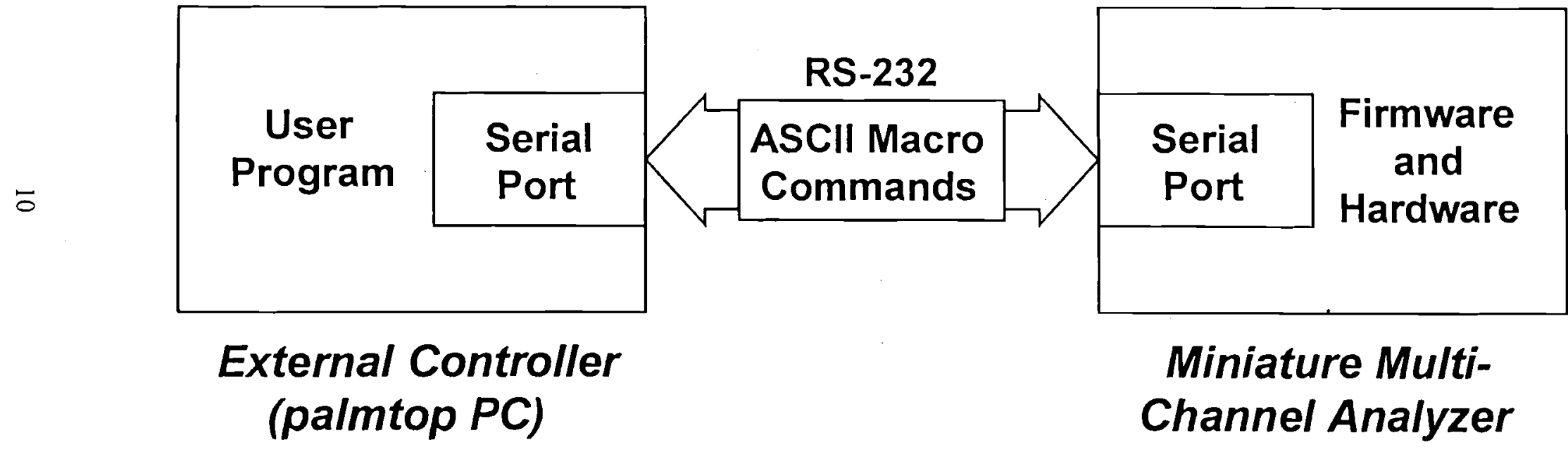

Figure 3.4. Interface schematic for the miniature data-acquisition hardware package (courtesy of the Los Alamos National Laboratory). 


\subsection{Field Demonstration Results}

\subsection{Fernald Incinerator Site}

During fiscal-year 1994, PNL participated in a Fernald-hosted effort to evaluate field screening tools that acquire high-resolution information about the distribution of uranium contamination in surface soils efficiently and cost effectively. Extensive soil sampling and laboratory analyses were performed by Fernald personnel in support of this effort, allowing direct comparison of the evaluated technologies. The site selected for investigation (Figure 4.1) was the large field north of Fernald's incinerator facility (approximately $12,000 \mathrm{~m}^{2}$ total area), where the primary source of surface contamination is stack emissions from incinerated uranium-contaminated combustibles (Schilk et al. 1993). The emissions were deposited on the soil by the prevailing southwesterly winds.

During field analysis, the sensor package was suspended above the soil surface and counts were initiated for 10 to 15 minutes at each sample location. Two soil-to-detector distances -- $15 \mathrm{~cm}$ and $100 \mathrm{~cm} \mathrm{--}$ were established to evaluate the effect of pseudo-collimation on spatial resolution. Moving the sensor closer to the ground effectively decreases the total area viewed, de-emphasizing spatial heterogeneities caused by contaminant and topographic irregularities.

Sensor reproducibility was investigated by revisiting two specific field locations (standard plots \#1 and \#2) throughout the course of the demonstration, and comparing detector output as a function of time and environmental conditions. This procedure allowed PNL investigators to correct daily results, when necessary, for any precipitation-induced discrepancies in the sensor response caused by intermittent rainfall. Knowledge of this phenomenon allowed investigators to scale the in situ gamma-ray spectrometer responses at other field locations monitored throughout each day to generate results based on dry-soil conditions.

Scaled measurements from this demonstration were modelled with a commercial geostatistical software package. Activity contour maps were generated showing observed decreases in uranium activity levels as a function of increasing distance between the deposition site and the incinerator (Figures 4.2 and 4.3). These maps are consistent with site historical information, which indicates that contaminated particles originating from the incinerator stack were transported to the north and east across the field site. Further support for the in situ gamma-ray spectrometry results were provided by concurrent surface-soil analyses, which led to a qualitatively similar contour map (Figure 4.4). Quantitatively, the results from these three measurements are not equivalent because of the spatial heterogeneity of the source and the differences in the sample area for each methodology (100-cm-high gamma-ray sensor sample area $>15-\mathrm{cm}$-high gamma-ray sensor sample area $>$ soil-sample area). Hence, actual activity values at each location are highest for the soil samples, lowest for the $100-\mathrm{cm}$ results, and intermediate with regard to the $15-\mathrm{cm}$ results. Despite these quantitative discrepancies, the combined data from these three measurements exhibit good to moderate correlation (Figures 4.5 through 4.7), lending support and credence to the use of the in situ gamma-ray spectrometry package for field measurements of surface activity under various environmental conditions. 


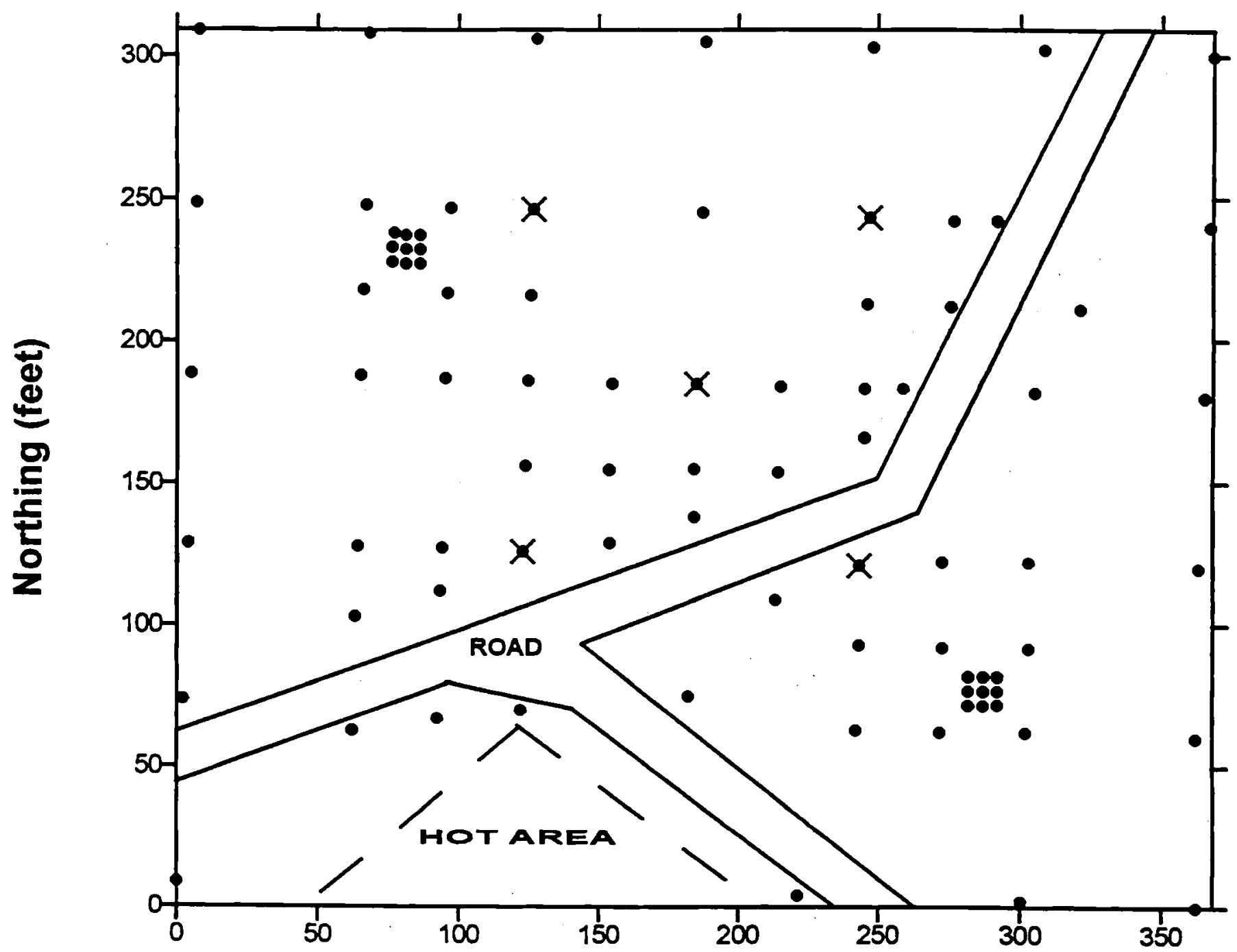

Easting (feet)

Figure 4.1. Base map for the field demonstration area, directly north of the Fernald incinerator area. Sample sites are indicated by solid circles; southernmost row of data points (discontinuous due to the presence of the exclusion area) are equivalent to those found immediately north of the northern fenceline surrounding the incinerator site as identified in Schilk et al. 1993a. The approximate location of the actual incinerator stack is: easting $=+50$ feet, northing $=-50$ feet. Crosses indicate samples that were cored for depth-distribution analyses. 


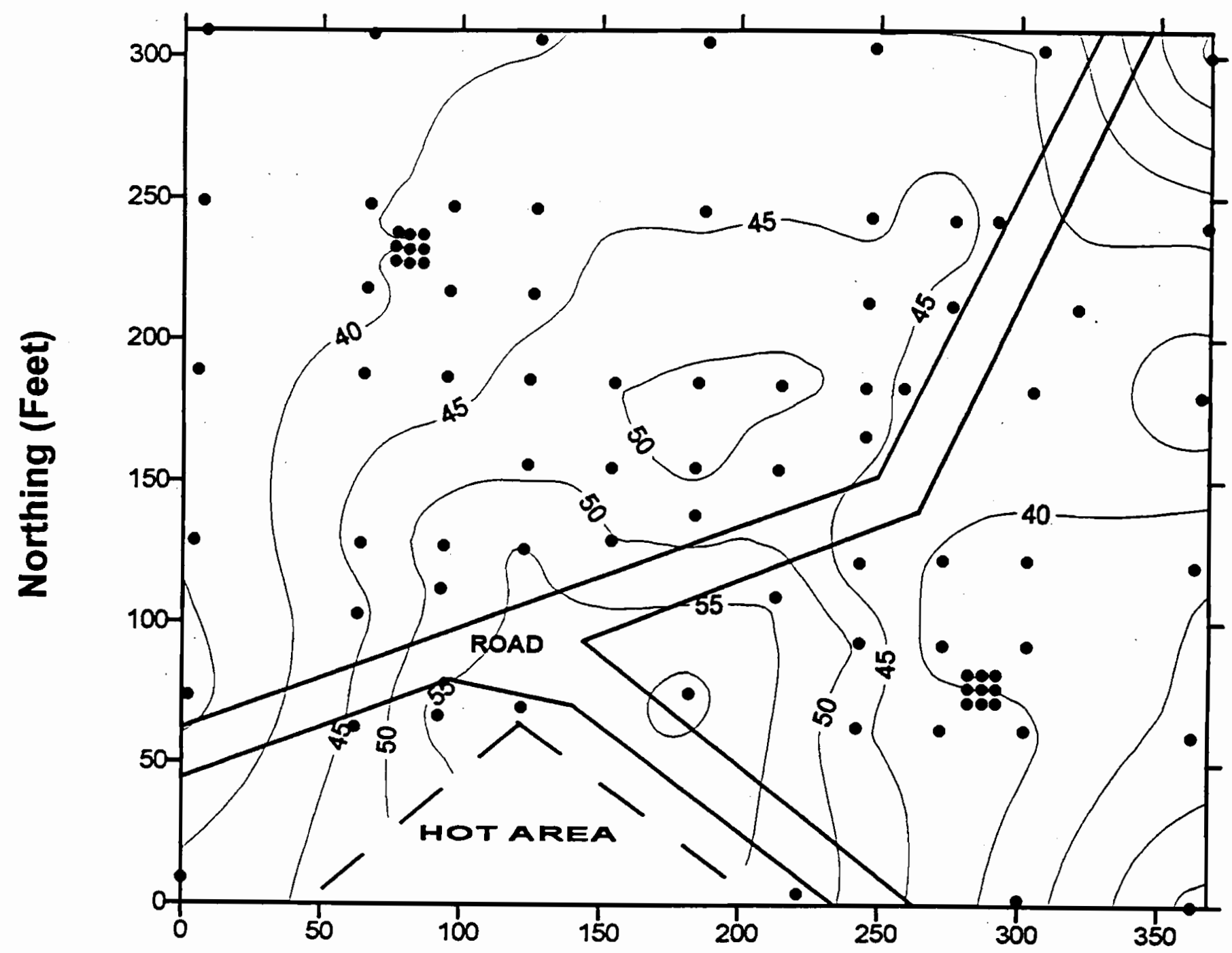

Easting (Feet)

Figure 4.2. Uranium surface activity contour map based on a geostatistical analysis of the $100-\mathrm{cm}$ in situ gamma-ray spectrometry measurements. 


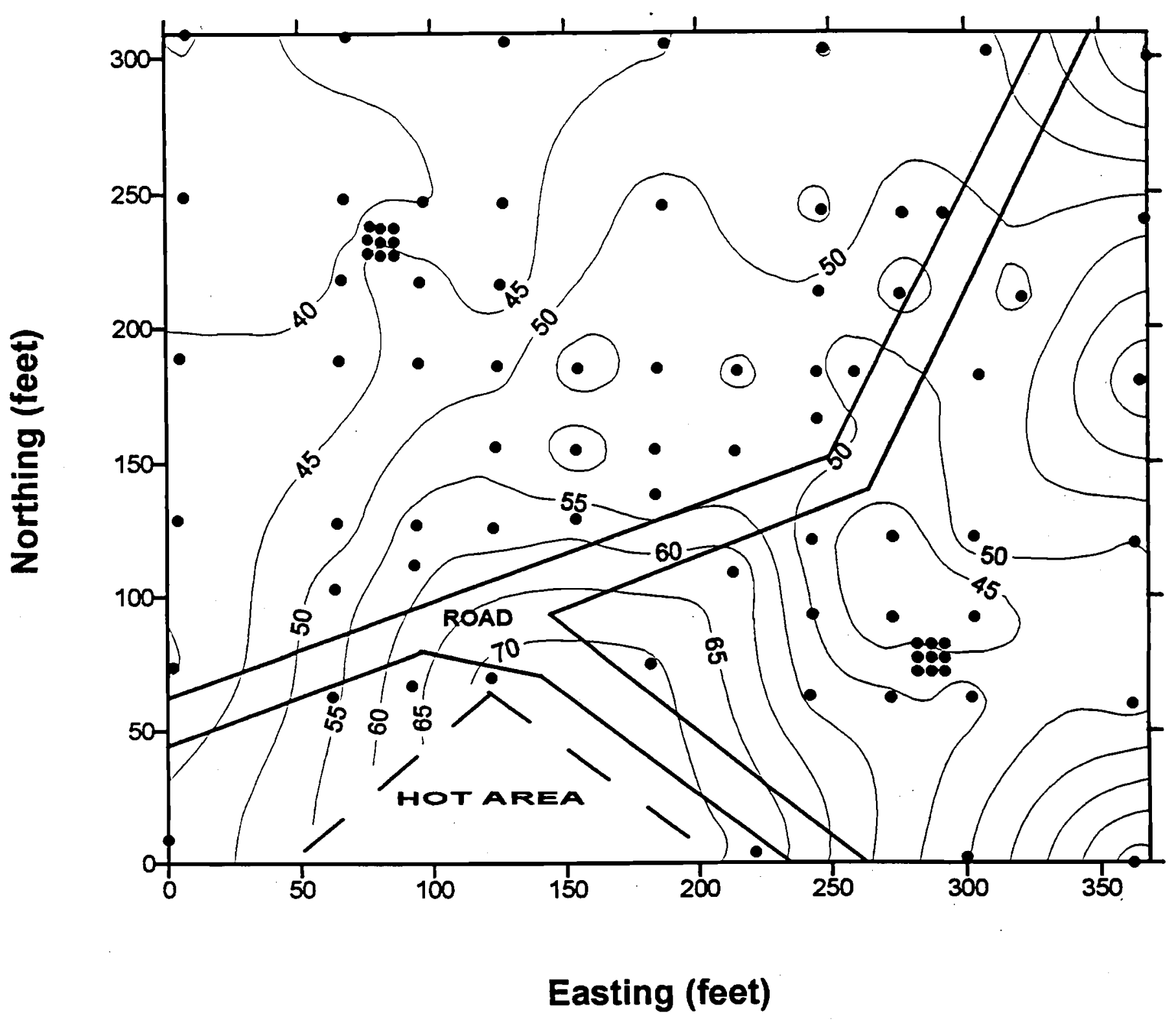

Figure 4.3. Uranium surface activity contour map based on a geostatistical analysis of the $15-\mathrm{cm}$ in situ gamma-ray spectrometry measurements. Qualitatively, these results are consistent with Figure 6. See text for explanation of quantitative differences. 


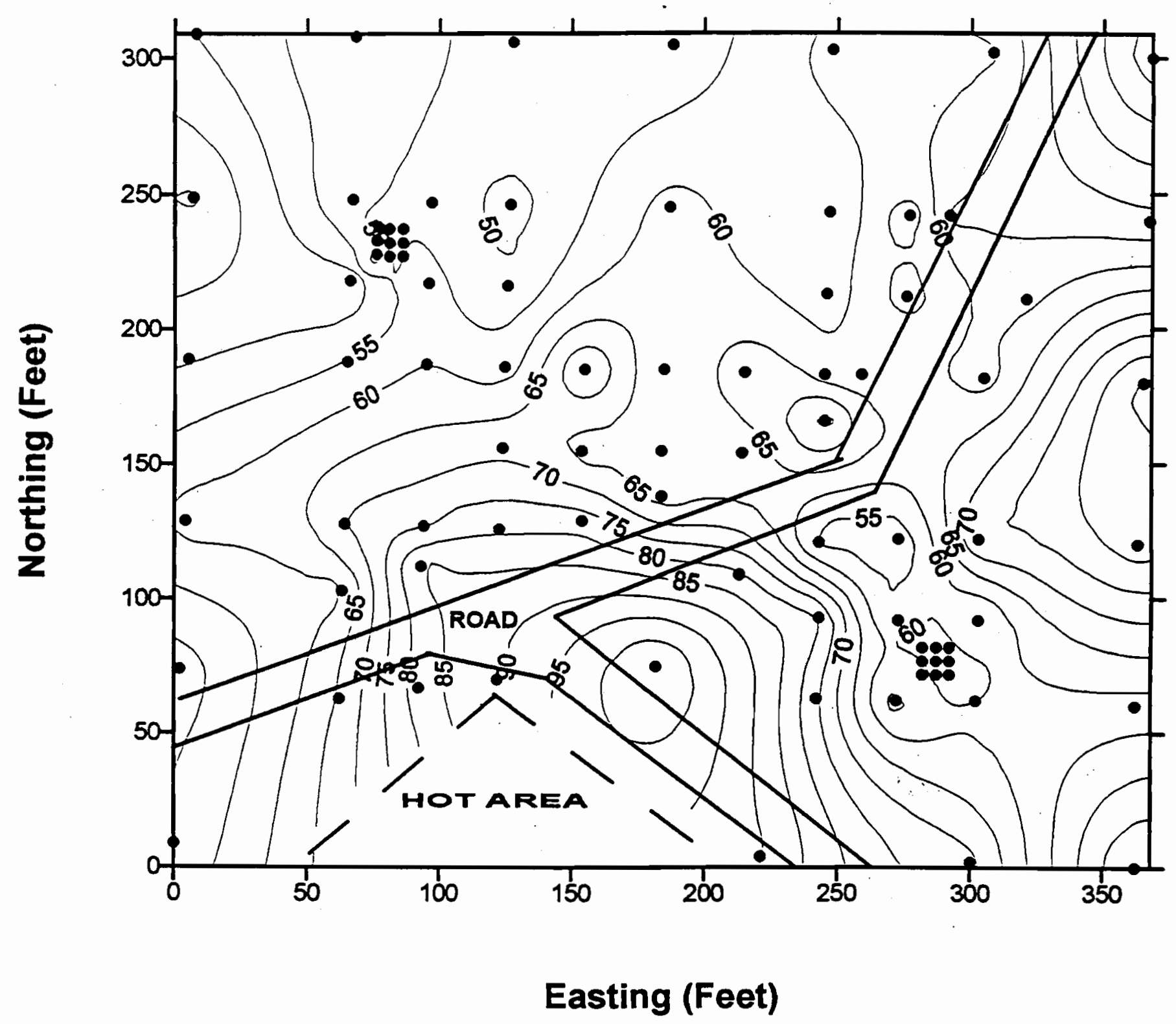

Figure 4.4. Uranium surface activity contour map based on a geostatistical analysis of the hand sample/laboratory analysis measurements. Qualitatively, these results are consistent with Figure 6. See text for explanation of quantitative differences. 


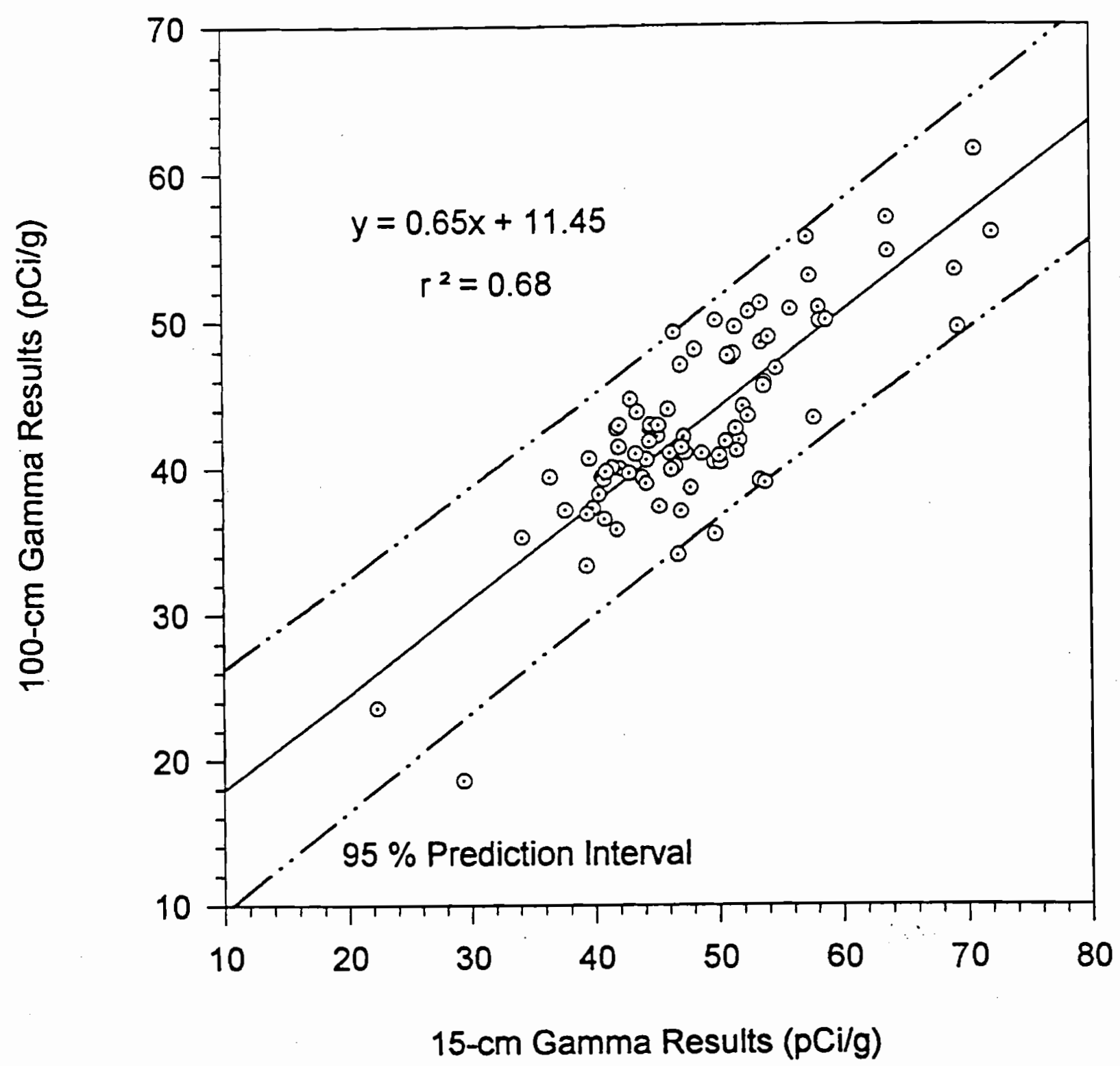

Figure 4.5. Correlation plot comparing the $100-\mathrm{cm}$ and $15-\mathrm{cm}$ in situ gamma-ray measurements. The correlation coefficient is moderate, indicating that the results tend to be mutually supportive. 


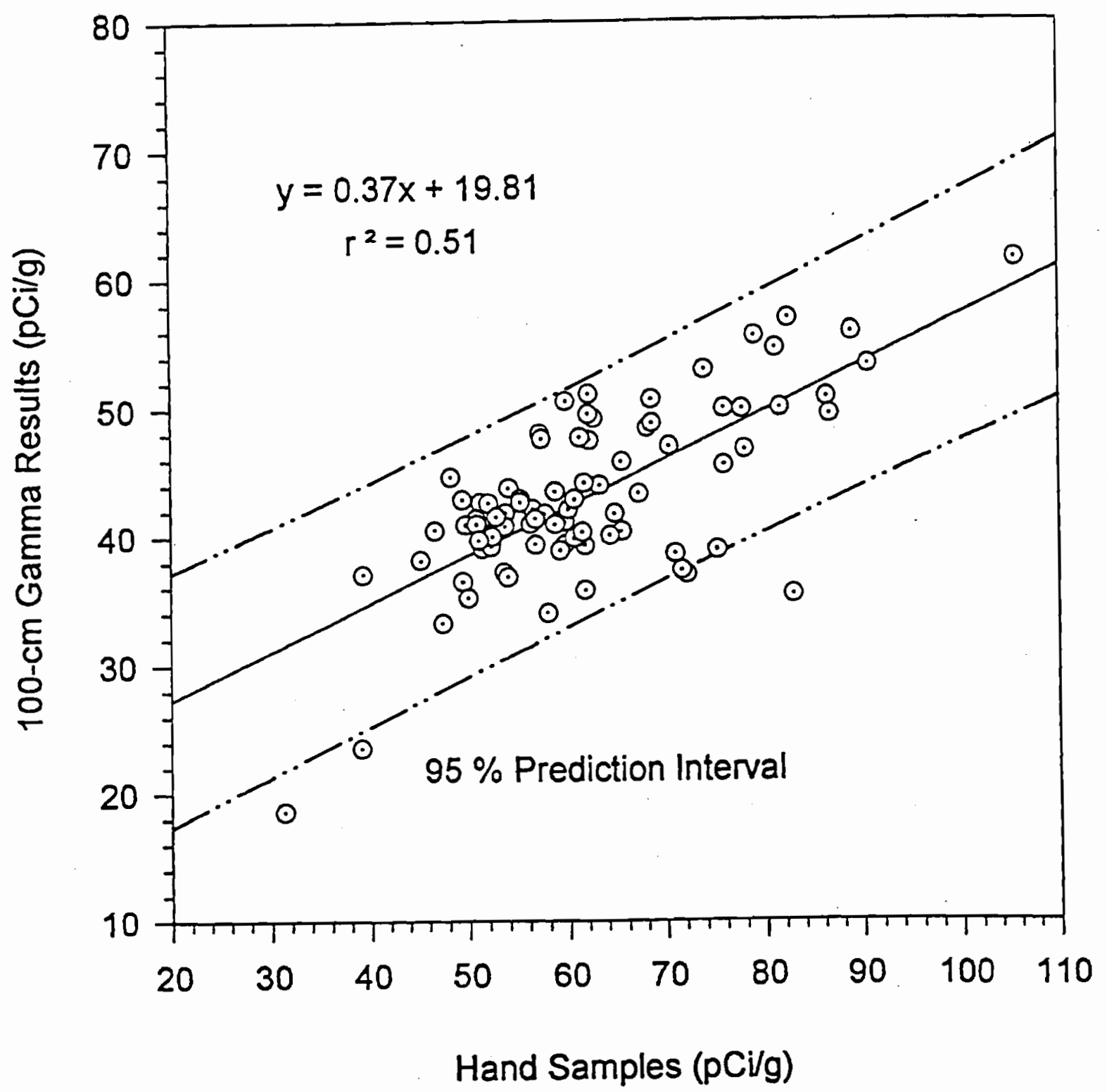

Figure 4.6. Correlation plot comparing the $100-\mathrm{cm}$ in situ gamma-ray measurements and the hand sample/laboratory analysis measurements. 


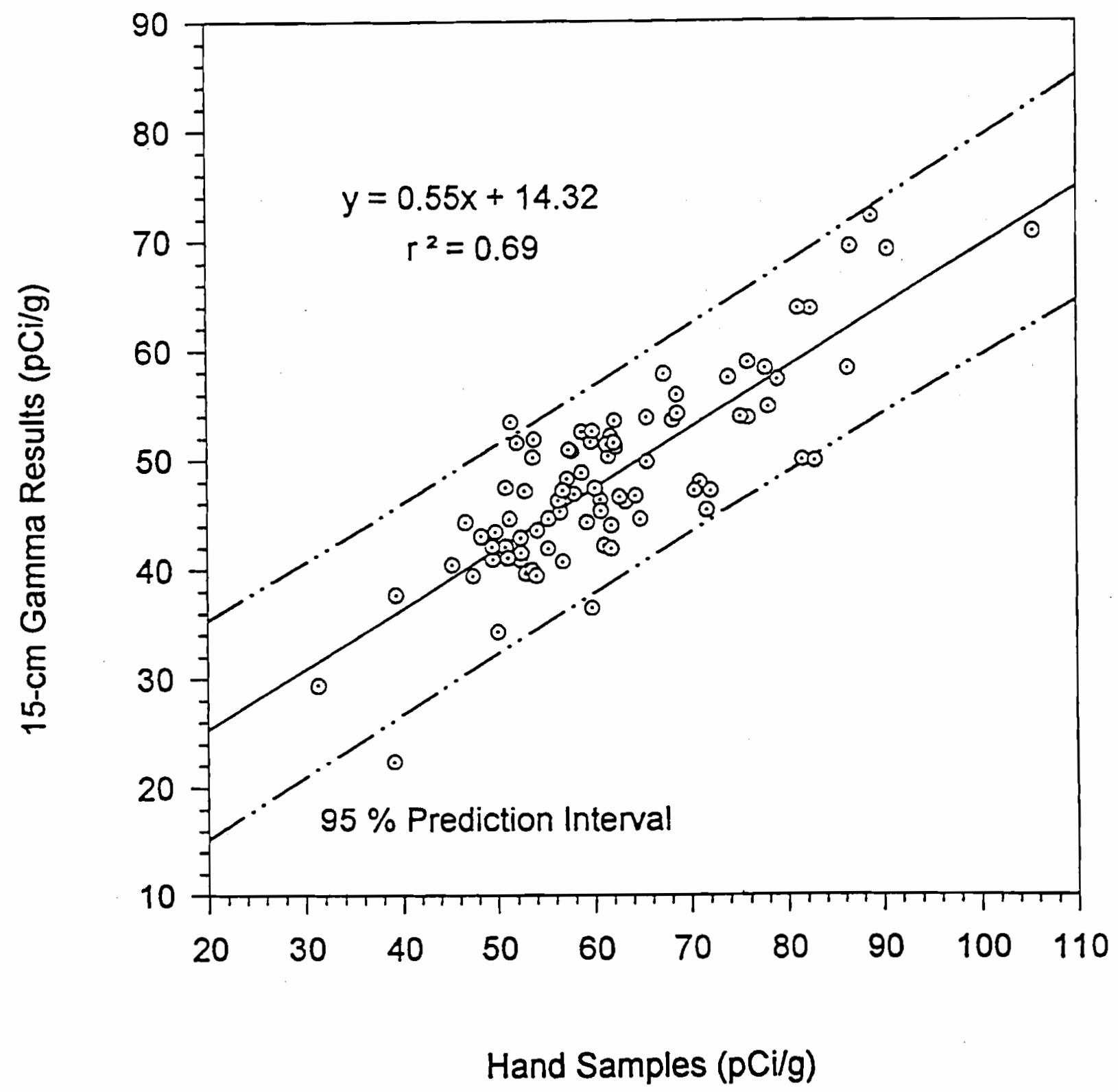

Figure 4.7. Correlation plot comparing the $15-\mathrm{cm}$ in situ gamma-ray measurements and the hand sample/laboratory analysis measurements. 


\subsection{St. Louis Airport Site (SLAPS)}

An additional field study was performed at SLAPS near St. Louis, Missouri, in support of the Ames Laboratory's Expedited Site Characterization demonstration. This facility was used in the late 1940s to store residues from nearby uranium ore processing, including barium sulfate cake, pitchblende and other radiumbearing materials, and contaminated scrap items.

During the second week in September, 1994, a number of sample locations within this facility were monitored with the improved in situ gamma-ray spectrometry system. Although soil samples were gathered at that time, assay results have not yet been provided to PNL for comparison with field analyses. 


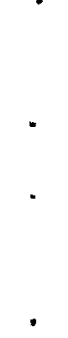




\subsection{System Capability for Depth-Distribution Determination of Contaminants}

A radionuclide-contaminated site must be completely characterized in three dimensions to establish remediation protocols. Without, at least, a first-order indication of the depth distribution of the contaminants, remediation activities run the risk of concurrently removing enormous quantities of uncontaminated soils. This will, at best, lead to additional cost and effort in terms of removal, transport, and storage, and, at worst, serve to dilute and disseminate the excavated contamination within a larger volume of clean material. The traditional means of determining subsurface activity levels is to drill or core target soils, transfer the contaminated samples to a laboratory, and perform various analytical techniques. This expensive and timeconsuming process tends to generate a significant amount of secondary mixed waste. Such pitfalls could be avoided with non-intrusive technologies that measure subsurface radioactivity.

It was proposed that in situ gamma-ray spectrometry be evaluated for its ability to determine vertical activity distributions by observing the multi-photon flux from a three-dimensional source (e.g., ${ }^{238} \mathrm{U}$ and its equilibrium daughters) ${ }^{\prime}$, and comparing these empirical results with those generated from a complex model that incorporates solid geometry factors, soil- and air-attenuation coefficients, detector efficiency as a function of photon energy and angle of incidence, individual photon branching ratios, and selected vertical distributions. Modelled results from a few common depth distributions (Table 5.1) appear to support the concept that the quantitative differences between such scenarios are distinguishable.

Table 5.1. Calculated intensity ratios for selected gamma rays from the ${ }^{238} \mathrm{U}$ decay chain.

\begin{tabular}{|l|c|c|c|c|}
\hline $\begin{array}{c}\text { Ratio of } \\
\text { Gamma-Ray } \\
\text { Intensities }\end{array}$ & $\begin{array}{c}\text { Exponential } \\
\text { (10-cm relax- } \\
\text { ation length) }\end{array}$ & Uniform & Planar & $\begin{array}{c}\text { Buried 10 cm } \\
\text { (uniform) }\end{array}$ \\
\hline $63-\mathrm{keV} / 93-\mathrm{keV}$ & 0.64 & 0.62 & 0.72 & 0.13 \\
\hline $63-\mathrm{keV} / 1001-\mathrm{keV}$ & 8.6 & 6.9 & 14.3 & 0.48 \\
\hline $93-\mathrm{keV} / 1001-\mathrm{keV}$ & 13.3 & 11.1 & 20.1 & 1.9 \\
\hline
\end{tabular}

\subsection{Fernald Incinerator Site}

To test the hypothesis that in situ gamma-ray spectrometry could provide a first-order indication of the depth distribution of uranium in the field, spectra from five separate locations at the Fernald facility (see Figure 4.1) were acquired and the data reduced. The vertical distributions at each of these sites was predicted, based on its gamma-ray spectrum, and compared to the modelled results. Unfortunately, conditions were not optimal and it is suspected that the final results were compromised by considerable standing water and saturated conditions caused by frequent, heavy rains (a factor that was not addressed by

'It is critical that the radionuclide in question (or its decay chain) emit photons of disparate energies, since the theory is based on the variable effect of soil attenuation on high-and low-energy photons as a function of depth. 
the computer model) $)^{2}$. Ground truth was provided by soil cores collected by Fernald personnel some weeks after the original field effort.

Table 5.2 lists the inter-photon ratios observed by the in situ gamma-ray sensor and Figure 5.1 is a plot of the soil-core results for the five target locations. The field gamma-ray results suggest a set of distributions that closely resemble a uniform source, whereas the core analyses indicate pseudo-exponential behaviors with activity levels that decrease rather slowly with depth (i.e., with relaxation lengths ${ }^{3}$ between 5 and 7 centimeters). Although these results are not in complete agreement, it is believed that the differences are due to the effects of surface soil moisture at the time of the gamma-ray field measurements. The presence of water in the upper soil column would tend to attenuate the lower-energy photons to a greater extent, decreasing their observed intensities with respect to the higher-energy photons. This would skew the observed low-to-high intensity ratios toward the modelled uniform distribution. In the future it is recommended that this technique be used only under dry-soil conditions as assumed by the computer model.

Table 5.2. Observed gamma-ray intensity ratios for five locations north of the Fernald incinerator site (refer to Figure 4.1).

\begin{tabular}{|l|c|c|c|c|c|}
\hline $\begin{array}{c}\text { Ratio of } \\
\text { Gamma-Ray } \\
\text { Intensities }\end{array}$ & NW & NE & SW & SE & Central \\
\hline $63-\mathrm{keV} / 93-\mathrm{keV}$ & 0.61 & 0.72 & 0.62 & 0.58 & 0.59 \\
\hline $63-\mathrm{keV} / 1001-\mathrm{keV}$ & 5.0 & 5.5 & 5.4 & 4.4 & 5.3 \\
\hline $93-\mathrm{keV} / 1001-\mathrm{keV}$ & 8.2 & 7.7 & 8.7 & 7.6 & 9.1 \\
\hline
\end{tabular}

\subsection{St. Louis Airport Site (SLAPS)}

Although analytical data from soil cores were not available at the time of this writing, preliminary field results may be compared with site historical data to evaluate the efficacy of the in situ gamma-ray methodology for developing a first-order indication of uranium depth distribution under unusual conditions. It is known from site records that following demolition and burial of contaminated structures, and removal of radionuclide-bearing containers, the entire area was covered with up to a 0.9 -meter layer of clean topfill as shielding. In addition to providing a means of attenuating the radiation emitted from the buried uranium, this soil served to retain a majority of the radon decay daughters produced in the underlying material.

${ }^{2}$ The actual depth of water saturation was not determined at the time of the in situ measurements, but this phenomenon certainly led to a situation in which the bulk attenuation properties of the soil column were no longer constant (or entirely consistent with the model).

${ }^{3}$ The relaxation length is the amount of attenuator that decreases the activity level by a factor of $\mathrm{e}^{-1}$. 


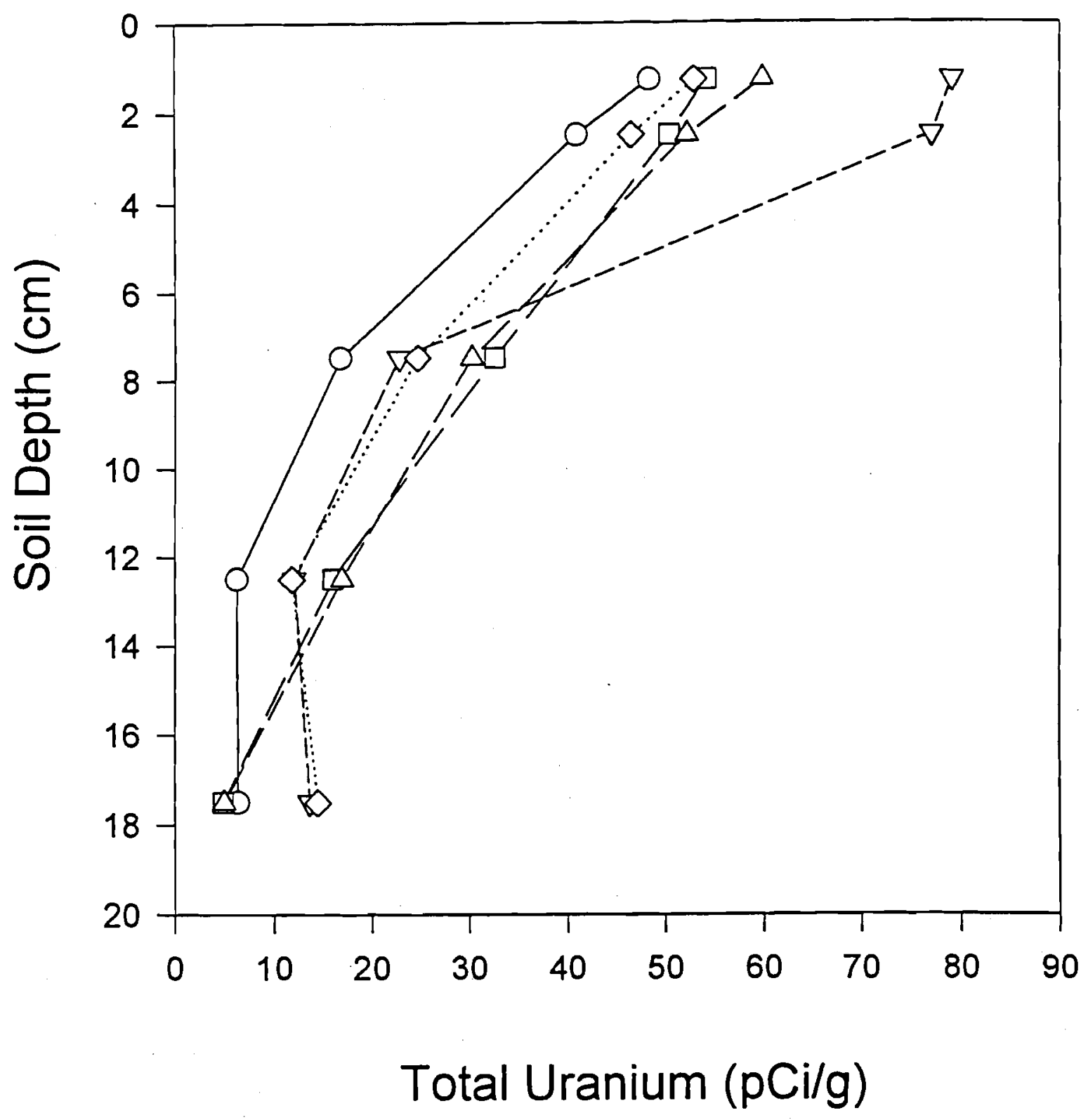

Figure 5.1. Measured uranium depth distributions for the five cored samples (refer to Figure 5 for locations). Legend: circle - NW point, square - NE point, triangle (down) - SW point, diamond SE point, triangle (up) - central point. 
Preliminary results corroborate the presence of a buried source at this abandoned facility. It is seen from the measure of ${ }^{214} \mathrm{Bi}$ activity, a multiple-photon-emitting radionuclide and decay daughter of ${ }^{238} \mathrm{U}$, that higher-energy photons lead to larger effective activities when one makes the assumption of a uniform distribution in the data-reduction process (Figure 5.2). Were this truly a uniformly distributed source, photon energy versus ${ }^{214} \mathrm{Bi}$ activity shown in Figure 5.2 would exhibit a straight line with a slope of zero; however, the actual slope indicates that the contaminant is overlain by a relatively clean overburden. First-order information such as this, obtained quickly and non-intrusively, is useful for establishing remedial protocols at previously uncharacterized sites.

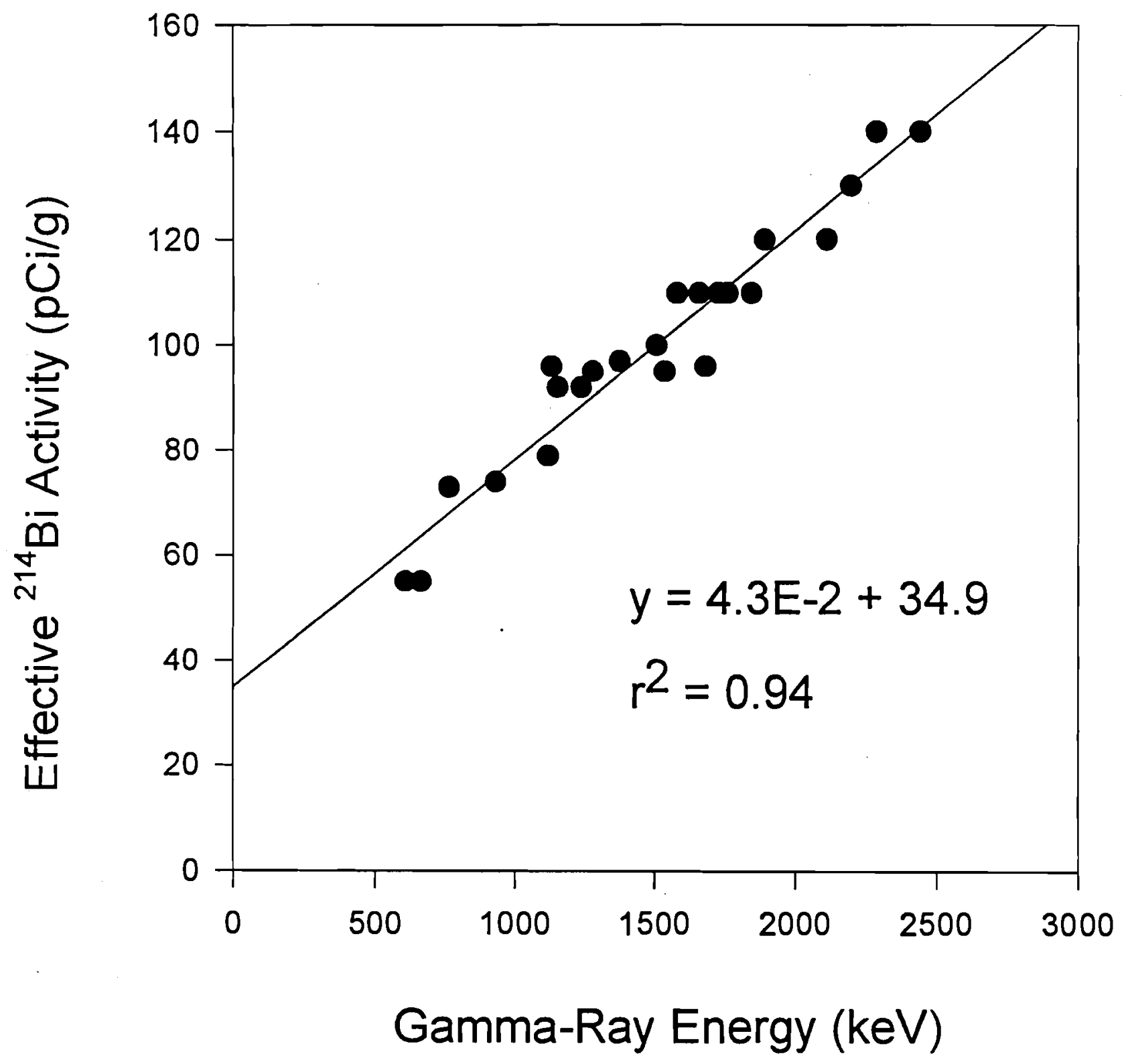

Figure 5.2. Plot of effective uranium activity as a function of photon energy when a uniformly distributed source was assumed. Increasing effective activity with higher photon energies implies a buried source. 


\subsection{Summary}

This document summarizes fiscal-year 1994 efforts at PNL to fulfill requirements for TTP \#321103 (project \#19307). These requirements included (a) developing a user-friendly software package for reducing field-acquired gamma-ray spectra, (b) constructing an improved data-acquisition hardware system for use with high-purity germanium detectors, (c) ensuring readiness to conduct field mapping exercises as specified by the sponsor, (d) evaluating the in situ gamma-ray spectrometer for the determination of uranium depth distribution, and (e) documenting these efforts. All requirements have been successfully completed and documented. Under the direction and auspices of the DOE's Uranium in Soils ID, PNL constructed a complete in situ system (composed of a commercially available n-type high-purity germanium sensor, an inhouse-developed data-reduction software code, and a miniaturized data-acquisition hardware unit), demonstrated and evaluated this package in various field scenarios, and provided this summary document. 



\subsection{References}

Halbig, J.K., S.F. Klosterbuer, P.A. Russo, J.K. Sprinkle, M.M. Stephens, and L.G. Wiig. 1993. "Recent Miniature MCA Technology Developments at Los Alamos." LA-UR-93-1728, Los Alamos National Laboratory, Los Alamos, New Mexico.

Halbig, J.K., S.F. Klosterbuer, P.A. Russo, and J.K. Sprinkle, Jr.. 1994. "Advances in and Uses of GammaRay Field Instrumentation at Los Alamos." LA-UR-94-276, Los Alamos National Laboratory, Los Alamos, New Mexico.

ICRU. 1995. "Gamma-Ray Spectrometry in the Environment." ICRU Report 53. International Commission on Radiation Units and Measurements, Bethesda, Maryland.

Schilk, A.J., R.W. Perkins, K.H. Abel, and R.L. Brodzinski. 1993. "Surface and Subsurface Characterization of Uranium Contamination at the Fernald Environmental Management Site." PNL-8617, Pacific Northwest Laboratory, Richland, WA. 

Appendix A

Summary of SASEA Screens and Options 


\section{Title Screen}

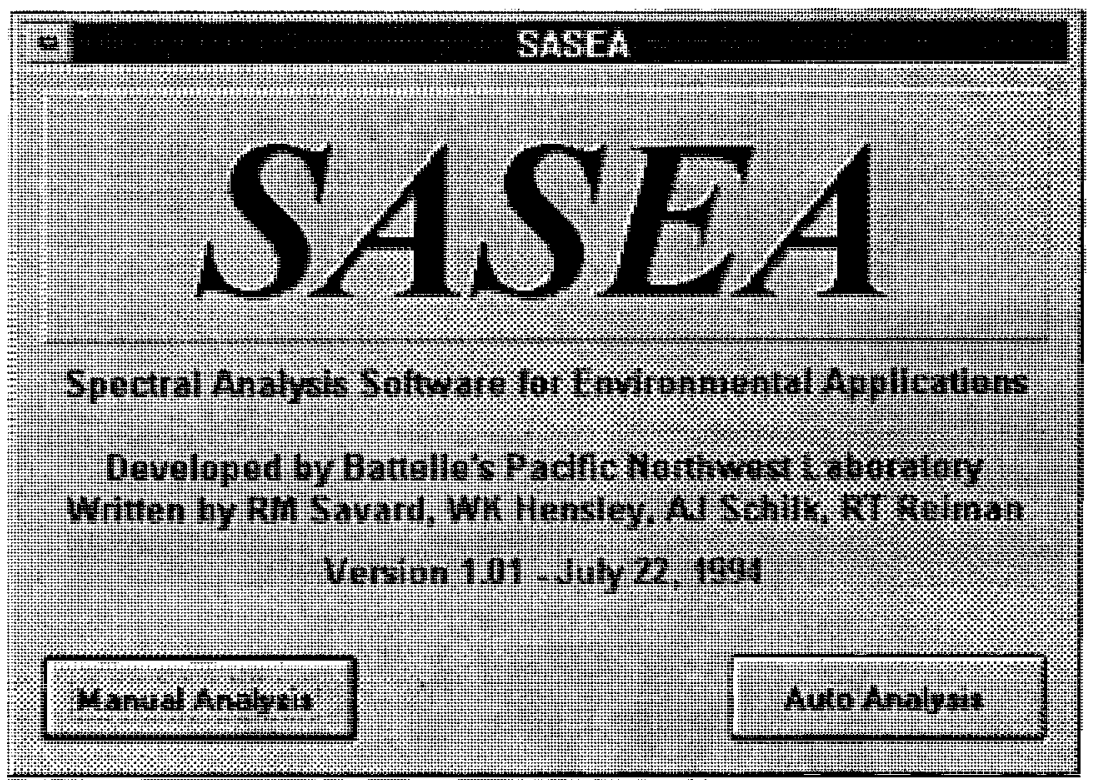

Pressing the Manual Analysis button takes you to the Manual Analysis screen (see below).

Pressing the Auto Analysis button takes you to the Auto Analysis screen (see below).

\section{Manual Analysis Screen}

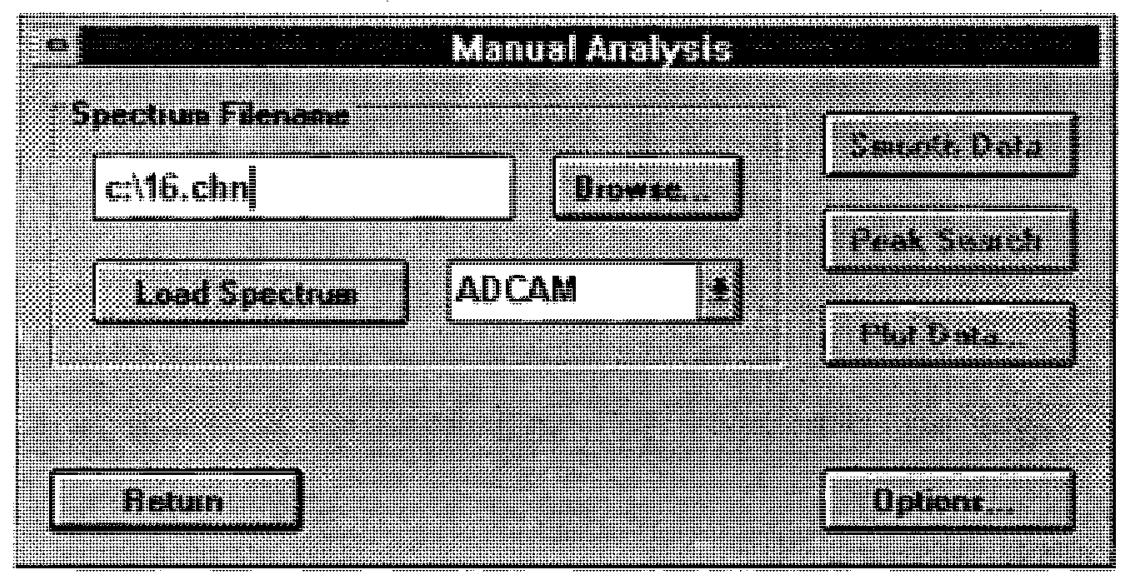

In the Combo Box, specify the format of the spectrum file you wish to analyze. Currently, you may choose either ADCAM or S100. Other file formats can be added as appropriate.

Enter the spectrum filename to analyze into the Text Box, and press the Load Spectrum button to load the spectrum file. Alternatively, press Browse... to choose the file from a file dialog box. 
Once a spectrum is loaded, you may press the Smooth Data button to smooth the data (using a 3 point moving average), the Peak Search button to find and identify the peaks, or the Plot Data button to bring up the Spectral Plot screen (see below).

Pressing the Options button takes you to the Options screen (see below).

Press $\underline{\text { Return }}$ to go back to the Title Screen.

\section{Auto Analysis Screen}

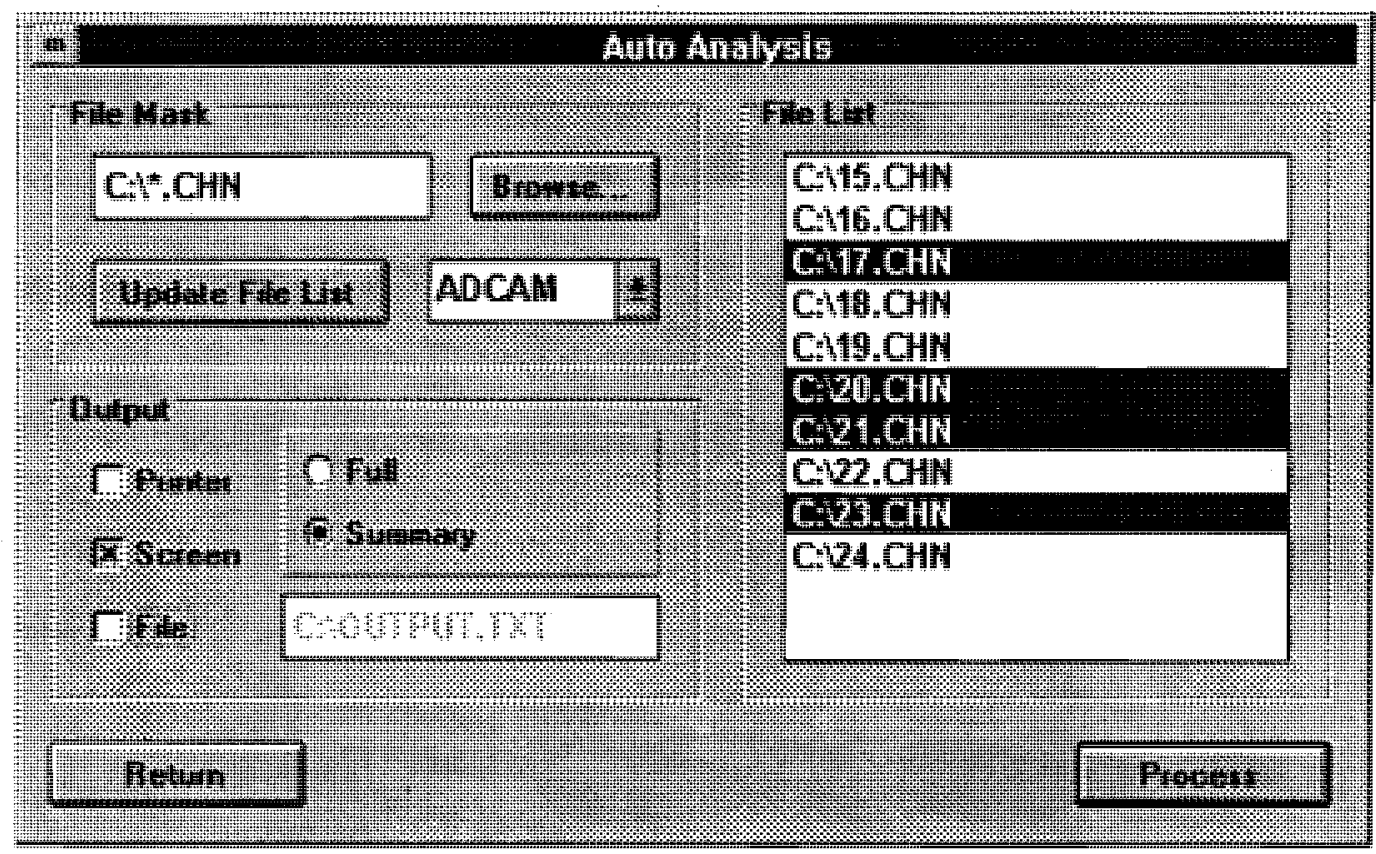

In the Combo Box, specify the format of the spectrum file you wish to analyze. Currently, you may choose either ADCAM or S100. Other file formats can be added as appropriate.

Enter a file mask into the text box, and press the Update File List button. All files matching the file mask will be shown in the File List. Alternatively, press Browse... to choose a file from a File Dialog box.

Select the file(s) you wish to analyze by highlighting them in the File List. To highlight more than one, hold down the CTRL key as you select the files.

In the Output box, choose where you want the output to go. You may choose the Printer, $\underline{\text { Screen, }}$ File, or any combination of them.

Selecting the Summary option will tell SASEA to print only the heading information of the analysis. The Full option will show all analysis information.

Press Process to begin the analysis process. 
Press $\underline{\text { Return }}$ to go back to the Title Screen.

\section{Spectral Plot Screen}

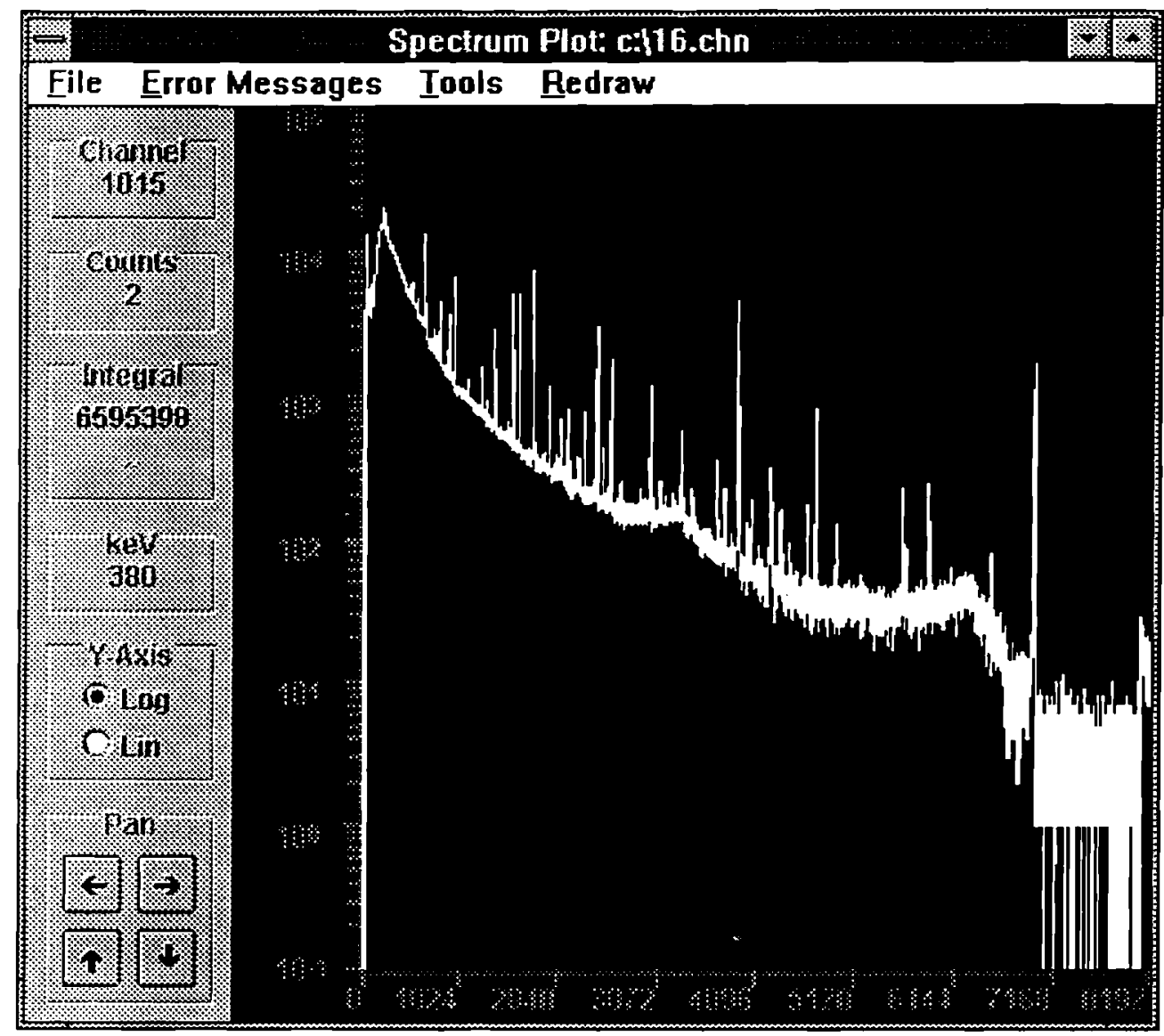

You may zoom in on any part of the graph by highlighting that region with the mouse cursor. You can zoom in multiple times. To zoom out, click the right mouse button.

While zoomed in, you can pan by pressing one of the arrow buttons on the left side of the window.

You can switch from a Logarithmic $y$-axis to a Linear $y$-axis at any time by clicking on the Lin option button. Click on the Log option button to change back to a Logarithmic y-axis.

The Channel, Counts, and $\mathrm{keV}$, which appear on the left side of the window, are updated continuously as you move your mouse pointer over the graph. They reflect the current mouse position.

Select the Exit command from the File menu to close this window.

The Error Messages menu controls whether or not error messages are displayed. (Error messages include "Selected region is too narrow to zoom in on," and the like.) 
The Tools menu controls whether or not the panel on the left side of the window is displayed. The Mini-Graph menu option controls the visibility of the mini-graph, a box that appears in the upperright corner when you have zoomed in on a region of the graph.

Selecting Redraw will redraw the graph.

\section{Options Screen}

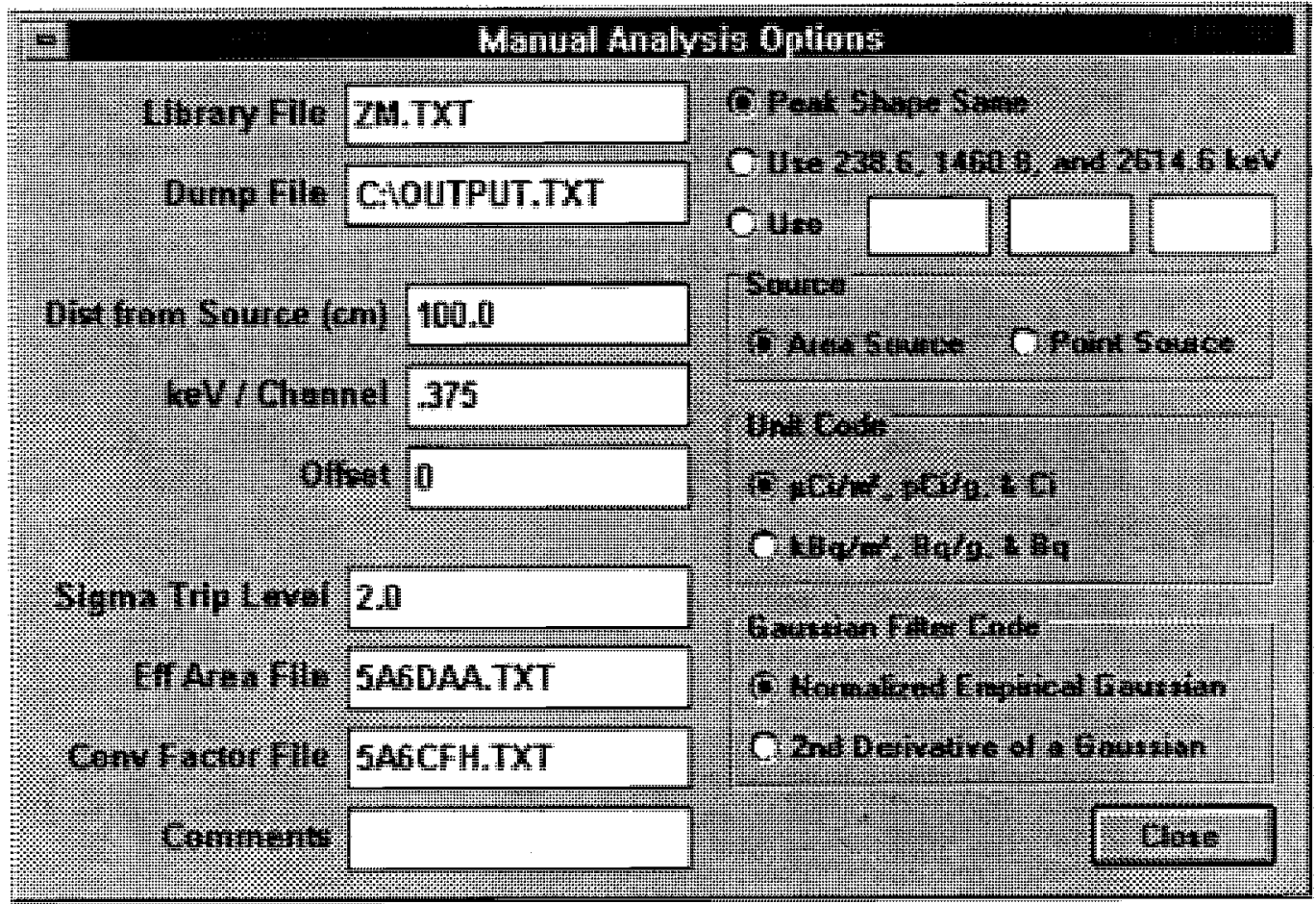

The Library File text box allows you to choose a specific peak library (e.g., natural isotopes only) to use in the peak search process.

The Dump File text box indicates your choice of output file name.

The Dist from Source text box is the source to detector distance in centimeters.

The $\mathrm{keV} /$ Channel text box and Offset text box indicate the calibration constants for the acquired spectra.

The Sigma Trip Level text box specifies the trip level for peak identification

The Eff Area and Conv Factor File text boxes identify the detector-specific files (obtained from the sensor calibration) necessary to reduce the spectral data.

The Comment box allows the input of text to appear at the top of the summary output. 
The Peak Shape and Use option buttons are intended to assist the operator in determining the best calibration/offset variables (see above).

The Source text box (Area vs. Point) establishes the areal extent of the observed source.

The Unit Code text box allows the choice of output units.

The Gaussian Filter Code options determine the peak search mode, i.e., the program may look for normalized gaussians or peaks in the 2 nd derivative of the raw spectrum. 


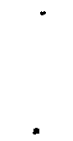




\section{Distribution}

No. of

Copies

Offsite

12 DOE/Office of Scientific and

Technical Information

DOE/Office of Technical

Development

C. W. Frank

Forrestal Building

U.S. Department of Energy

1000 Independence Ave SW

Washington, DC 20585

10 M. Malone

U.S. Department of Energy

EM-551, Trevion II

Washington, D.C. 20585-0002

\section{R.T. Reiman}

Technical Measurements Company

2888 Bluff Street

Suite 147

Boulder, CO 80301

J.K. Halbig

Los Alamos National Laboratory

Safeguards Assay Group, N-1

Los Alamos, NM 87545
No. of

Copies

Onsite

DOE Richland Operations Office

D. E. Trader

18

Pacific Northwest Laboratory
A. J. Schilk (5)
C. W. Hubbard
M. A. Knopf
K. H. Abel
D. E. Robertson
S. C. Slate (3)
Publishing Coordination
Technical Report Files (5) 


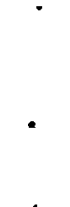

